\title{
Spatial coding defects of hippocampal neural ensemble calcium activities in the 3xTg-AD Alzheimer's disease mouse model
}

\section{Xiaoxiao Lin ( $\square$ xiaoxl3@uci.edu )}

University of California Irvine https://orcid.org/0000-0003-4488-6002

\section{Lujia Chen}

University of California Irvine

\section{David Baglietto-Vargas}

University of Malaga: Universidad de Malaga

\section{Qiao Ye}

University of California Irvine

\section{Frank M LaFerla}

University of California Irvine

\section{Douglas A. Nitz}

University of California San Diego

\section{Todd C. Holmes}

University of California Irvine

\section{Xiangmin $\mathrm{Xu}$}

University of California Irvine

\section{Research article}

Keywords: Alzheimer's Disease, aging, 3xTg-AD, in vivo imaging, calcium imaging, miniscope, freely behaving mice

Posted Date: May 26th, 2021

DOI: https://doi.org/10.21203/rs.3.rs-550358/v1

License: (c) (i) This work is licensed under a Creative Commons Attribution 4.0 International License. Read Full License 
Title: Spatial coding defects of hippocampal neural ensemble calcium activities in the 3xTg-AD Alzheimer's disease mouse model

Xiaoxiao Lin ${ }^{1 *}$, Lujia Chen ${ }^{1,6^{*}}$, David Baglietto-Vargas ${ }^{2,3 *}$, Qiao Ye ${ }^{1,6}$, Frank M LaFerla ${ }^{3}$,

Douglas A. Nitz ${ }^{4,7}$, Todd C. Holmes ${ }^{5,7}$, Xiangmin $\mathrm{Xu}^{1,3,6-7 \#}$

1. Department of Anatomy and Neurobiology, School of Medicine, University of California, Irvine, CA 92697

2. Department of Cell Biology, Genetic and Physiology, Faculty of Sciences, Biomedical Research Institute of Malaga (IBIMA), Networking Research Center on Neurodegenerative Diseases (CIBERNED), University of Malaga, Malaga, 29071, Spain.

3. Institute for Memory Impairments and Neurological Disorder, University of California, Irvine, CA, 92697

4. Department of Cognitive Science, University of California, San Diego

5. Department of Physiology and Biophysics, School of Medicine, University of California, Irvine, CA 92697

6. Department of Biomedical Engineering, University of California, Irvine, CA 92697

7. Center for Neural Circuit Mapping, University of California, Irvine, CA 92697

${ }^{*}$ X.L., L.C. and D.B. contributed equally to this work.

\# Lead contact / Address all manuscript correspondence to: Dr. Xiangmin Xu, Department of Anatomy and Neurobiology, School of Medicine, University of California, Irvine, CA 92697-1275 Tel: 949.824.0040 Email: xiangmix@hs.uci.edu

Author Email Addresses:

Xiaoxiao Lin: xiaoxl3@uci.edu; Lujia Chen: lujiac1@uci.edu; David Baglietto-Vargas: d.baglietto@uma.es; Qiao Ye: yeq4@uci.edu; Frank M LaFerla: laferla@uci.edu; Douglas A. Nitz: dnitz@ucsd.edu; Todd C. Holmes: tholmes@uci.edu

Running Title: Miniscope imaging in the AD model mouse Pages: 36

Figures: 5

Author contributions: X.L. and Q.Y. performed imaging, behavioral and immunostaining experiments. L.J., X.L., D.B., X.X., T.C.H., D.N. analyzed the data, prepared the figures and wrote the manuscript with the help from other authors. X.X. designed and oversaw the project. All authors read and approved the final manuscript.

\section{Acknowledgements}

This work was supported by US National Institutes of Health (NIH) grants (R01NS078434, R01NS104897 and RF1AG065675) to X.X. and D.N., R35 GM127102 to T.C.H..

\section{Statement of conflict of interests}

All authors disclose no conflict of interests for this work 


\section{Abstract}

\section{Background}

Alzheimer's disease (AD) causes progressive age-related defects in memory and cognitive function, and has emerged as a major health and socio-economic concern in the US and worldwide. To develop effective therapeutic treatments for $A D$, we need to better understand the neural mechanisms by which AD causes memory loss and cognitive deficits.

\section{Methods}

Here we examine large scale hippocampal neural population calcium activities imaged at single cell resolution in a triple-transgenic Alzheimer's disease mouse model (3xTg-AD) that presents both amyloid plaque and neurofibrillary pathological features along with age-related behavioral defects. To measure encoding of environmental location in hippocampal neural ensembles in the 3xTg-AD mice in vivo, we performed GCaMP6-based calcium imaging using head-mounted, miniature fluorescent microscopes ("miniscopes") on freely moving animals. We compared hippocampal CA1 excitatory neural ensemble activities during open-field exploration and track-based route-running behaviors in age-matched AD and control mice at young (3 - 6.5 months old) and old (18 - 21 months old) animals.

\section{$\underline{\text { Results }}$}

3xTg-AD CA1 excitatory cells display significantly higher calcium activity rates compared with Non-Tg controls for both the young and old age groups during spatial exploration, suggesting that in vivo enhanced neuronal calcium ensemble activity is a disease feature. Increased ages are correlated with decreased neural calcium activity rates across genotypes. CA1 neuronal populations of 3xTg-AD mice show lower spatial information scores compared with control mice. Spatial firing of CA1 neurons of old 3xTg-AD mice also displays higher sparsity and spatial coherence, indicating less place specificity for spatial representation. We find locomotion significantly modulates the amplitude of hippocampal neural calcium ensemble activities in 3xTgAD mice, but not in non-transgenic controls during open field ambulatory movements. 


\section{$\underline{\text { Conclusions }}$}

Our data offers new and comprehensive information about age-dependent neural circuit activity changes in this important AD mouse model, and provides strong evidence that spatial coding defects in the neuronal population activities are associated with AD pathology and ADrelated memory behavioral deficits.

Keywords: Alzheimer's Disease, aging, 3xTg-AD, in vivo imaging, calcium imaging, miniscope, freely behaving mice 


\section{Background}

Alzheimer's disease (AD) is an incurable age-related progressive neurodegenerative disorder. Individuals diagnosed with AD undergo a progressive loss of memory, cognition, language skills and personality traits (2020; Weller and Budson, 2018). Currently, the neural mechanisms by which AD causes memory loss and cognitive deficits remain poorly understood. However, recent imaging studies from clinical and animal models imply that neuronal dysfunction and functional disruption of neuronal circuits in the brain strongly contribute to memory deficits in AD cases (Busche and Konnerth, 2016; Frere and Slutsky, 2018). As the number of AD cases steadily increase each year and current treatments are only palliative, further understanding of AD-related neural mechanisms is needed for the development of meaningful new treatment strategies for improving memory and prolonging healthy cognitive function (Bast et al., 2017; Harris et al., 2020; Selkoe, 2002).

One of the major brain areas affected in AD patients is the hippocampal formation. This brain region and its connections with several cortical areas play essential roles in spatial cognition and episodic memory processes. Recent studies in humans, and supported by animal models, show that the CA1 projection to the subiculum (SUB) area is significantly affected in AD (Busche et al., 2012; Lerdkrai et al., 2018). CA1 and SUB regions project to diverse cortical areas that include the entorhinal, retrosplenial and perirhinal cortex and these cortical areas are implicated in multiple forms of spatial cognitive and memory processes (Cembrowski et al., 2018; Naber et al., 2000; Xu et al., 2016). Moreover, alterations in the balance of neural circuit excitation / inhibition have been found in $A D$, which may underlie dysfunctional circuit changes observed in AD cases (Busche et al., 2012). Several studies in AD animal models have shown that pathologically associated neural activity is a major feature in these models. Interestingly, neuronal hyper-activity or hypo-activity has been described in the vicinity of amyloid plaque formation (Busche et al., 2012; Grienberger et al., 2012; Palop and Mucke, 2016; Yang et al., 
2018), suggesting that aberrant neuronal activity changes are associated with the defects in cognitive processes in $A D$ brains.

Here, we used in vivo GCaMP6-based calcium imaging with head-mounted, miniature fluorescent microscopes ("miniscopes") on freely moving animals (Cai et al., 2016; Chen et al., 2013; Ziv et al., 2013) to study CA1 neural circuit ensemble coding associated with AD-related memory impairments in a triple-transgenic Alzheimer's disease mouse model (3xTg-AD). Headmounted miniscope imaging enables us to longitudinally examine hundreds of brain cells in action at single cell resolution, as the animal freely explores environments.

The 3xTg-AD mouse model contains three mutations in homologous mouse genes associated with human familial Alzheimer's disease (APP Swedish, MAPT P301L, and PSEN1 M146V), and displays age-dependent plaque and tangle pathology. Extracellular amyloid beta $(A \beta)$ deposits appear by 6 months and become progressively more extensive by 12 months in $3 x T g-A D$ mouse brains. Although tau pathology is not robustly observed in $3 x T g-A D$ mice by six months age, aggregates of conformationally-altered and hyperphosphorylated tau are detected in the hippocampus of 3xTg-AD mice by 12-15 months (Billings et al., 2005; Oddo et al., 2003).

The 3xTg-AD line reveals hippocampal spatial memory impairments at relatively earlier ages, even preceding plaque and tangle formation (Baglietto-Vargas et al., 2018; Oddo et al., 2003; Stimmell et al., 2019). We draw on a strong premise from the literature and our previous studies that memory-based behaviors rely on neural circuitry that are altered in aging and AD (Bast et al., 2017; Busche and Konnerth, 2016; Frere and Slutsky, 2018; Harris et al., 2020; Selkoe, 2002). Using large scale miniscope imaging of neural calcium activity, we studied the neural calcium ensemble activities in hippocampal CA1 in freely behaving 3xTg-AD mice and non-transgenic controls at young (3 - 6.5 months on average) and old (18 - 21 months old) ages. We find spatial coding defects in hippocampal neural calcium ensembles in the 3xTg-AD mouse model. This provides strong evidence that spatial encoding defects in hippocampal circuit ensemble activities are associated with AD-related memory behavioral deficits. 


\section{Methods}

\section{Animals}

All experiments were conducted according to the National Institutes of Health guidelines for animal care and use and were approved by the Institutional Animal Care and Use Committee and the Institutional Biosafety Committee of the University of California, Irvine. Female nontransgenic (Non-Tg) and 3xTg-AD mice at 3.6-6.5 and 18.5-21 months of age were used in this study. Our 3xTg-AD mice were provided by the La Ferla's lab and the MODEL-AD UCI center, The generation of the 3xTg-AD mice has been described previously (Belfiore et al., 2019; Oddo et al., 2003). Briefly, the 3xTg-AD model has been generated by co-microinjected two independent transgenes encoding human APPswe and the human TauP301L (both under the control of the mouse Thy1.2 regulatory element) into single-cell embryos harvested from homozygous mutant PS1M146V Knockin (PSI-KI) mice. We used the F1 mice from the cross of 129X1/SvJ (JAX reference 000691) with C57BL6 (JAX reference 000664) as non-transgenic controls. The mice had access to food and water in their home cages with lights maintained on a $12 \mathrm{~h}$ light/dark cycle.

\section{Viral injections}

In order to image in vivo calcium transients of hippocampal CA1 excitatory neurons, AAV1CamKII(0.4)-GCaMP6f was stereotaxically injected into the mouse brain region. First, the animal was anaesthetized under $2 \%$ isoflurane for 10 minutes with a $0.8 \mathrm{~L} / \mathrm{min}$ oxygen flow rate using an isoflurane tabletop unit (HME109, Highland Medical Equipment). The mouse was then head secured in a rodent stereotaxic (Leica Angle Two ${ }^{\mathrm{TM}}$ for mouse) with continuous $1.5 \%$ isoflurane anesthesia. An incision was made to expose the skull, and a small craniotomy was performed for the viral injection. The coordinates of Bregma and lambda were used as landmarks to determine the target brain region with the coordinates: anteroposterior (AP) $-1.94 \mathrm{~mm}$, lateromedial $(\mathrm{ML})$ $+1.40 \mathrm{~mm}$; dorsoventral (DV) $-1.35 \mathrm{~mm}$ (all values given relative to the bregma). The GCaMP- 
expressing virus (0.4 ul of AAV1-CaMKII(0.4)-GCaMP6f, $\left.2 \times 10^{11} \mathrm{GC} / \mathrm{ml}\right)$ was delivered into the target region at a rate of $20-30 \mathrm{nl} / \mathrm{min}$ with $10 \mathrm{~ms}$ pulse duration by a Picospritzer (General Valve, Hollis, NH). After injection, the glass needle stayed in the brain for 5 min to prevent backflow of the virus. A tissue adhesive (3M Vetbond, St. Paul, MN) was used to close the incision. Three weeks later, the mouse was implanted with a GRIN lens for miniscope imaging.

\section{Imaging experiment preparations}

The related mouse surgery has been described in our published study (Sun et al., 2019). All the animals were implanted with a GRIN lens for in vivo calcium imaging once they were recovered from AAV1-CaMKII-GCaMP6f injection. Following the same procedure of viral injection, animals were anesthetized under $2 \%$ isoflurane and placed in a heating pad with a setting temperature at 37C. Once applied $70 \%$ ethanol and Betadine on the shaved head, the skin tissue was opened. The connective tissue and tendons on the surface of the skull were removed by swab and fine forceps. The muscle was dissected from the edge of the skull by a scalpel. To enhance the stability of implantation and in vivo imaging quality, we used a bur (Meisinger, 1/4 Round Steel) to roughen the surface of skull and implant a skull screw far away from the implantation area. Saline was used to irrigate the skull, which can reduce the overheating caused by bur and clean up the skull. We marked a center point for craniotomy on the exposed skull (AP: $-2.3 \mathrm{~mm}, \mathrm{ML}:+1.75 \mathrm{~mm}$ ) and etched a $1-\mathrm{mm}$ radius cranial window surrounding the center point, which was allowed a 1.8mm diameter GRIN lens (Edmund Optics) to stabilize in it. The skull fragment was carefully removed with fine forceps and the exposed tissue was gently aspirated with a $27 \mathrm{G}$ flat needle. We then switched to a $29 \mathrm{G}$ flat needle until seeing the white striated tissue (corpus callosum) above CA1. We stop the aspiration when the hippocampus was exposed. We then attached the prepared lens holder to the stereotaxic apparatus and gently lowered the GRIN lens to the target depth (DV: $-1.55 \mathrm{~mm}$ ). A small amount of crazy glue was quickly applied nearby the GRIN lens to cover the exposed tissue. The 
adaptive spray was used to dry the crazy glue and stabilize the lens in a short time. A thick layer of dental cement (Lang Dental Manufacturing: 1304CLR) was used to secure the implant to the skull. We applied Kwik-Sil on the top of the lens to protect the lens from physical damage until the dental cement was dried. We wait for 2-3 weeks for the hippocampal tissue to recover from the surgery damage. A miniature epifluorescence microscope was used to check neural activity through a GRIN lens and to prepare for the placement of baseplate. The baseplate was stabilized by dental cement. We then attached a cap on the baseplate to prevent the damage of the lens caused by daily activity.

\section{Histology and immunochemical staining}

All animals were perfused after behavioral studies. $5 \mathrm{ml}$ of phosphate buffered saline (PBS) were used to push out blood in the brain and followed by $25 \mathrm{ml}$ PBS containing $4 \%$ paraformaldehyde. The perfused mouse brain was soaked in $4 \%$ paraformaldehyde for overnight and was switched into $30 \%$ sucrose in 1 X PBS until the brain sinks. In the next, the brain was frozen in dry ice and coronally sectioned in $30 \mu \mathrm{m}$ thickness on a microtome (Leica SM2010R, Germany). Half of the hippocampal sections were mounted for the checking of GRIN Lens implantation. The contralateral hippocampal sections without the removal of cortex were immunostained with various antibodies to identify the pathology of Alzheimer's Disease. To investigate the intracellular $A \beta$ accumulation and APP related products, we stained hippocampal sections with mouse anti- $\beta-$ Amyloid primary antibody (6E10, BioLegend, 1:500 dilution, Cat: 803010) and following by an Alexa Fluor 549 conjugated donkey anti-mouse secondary antibody (Jackson Immuno Research, 1:200 dilution). For tau staining, a mouse anti-AT8 tau antibody (Thermo Fisher, 1:250 dilution, Cat: MN1020) was used and followed by the same AF549 donkey anti-mouse secondary antibody. Sections were stained with DAPI and cover slipped for imaging.

The images of immunostained sections were acquired by a fluorescent BX61 Olympus microscope. In addition, AAV-expressing hippocampal sections were imaged with a confocal 
microscope (FV3000, Olympus) under a 20X objective lens. The acquired images were exported and performed using the software analysis tools.

\section{Behavioral experiments}

All the animals were handled and habituated with a head-mounted miniscope 5 min per day for at least one week before behavior experiments. Water restriction was conducted to motivate animals to move on the track and the reduction of body weights were controlled within $15 \%$.

Open arenas (circle and square box). After being well handled, animals were habituated in two open field arenas with a head-mounted miniscope for 4 consecutive days. Circle box ( $36 \mathrm{~cm}$ in diameter) and square box (26 cm in length) were adorned with various visual cues. On the first day, animals explored the circular environment for 10 minutes, then were transferred to the adjacent square box for another 10 minutes. On the second day, animals explored in each arena for 10 minutes as day one except exposing to the square arena first then to the circular box. Following the habituation in two boxes on four consecutive days, animals' behavioral trajectories and neuronal activities were recorded by miniscope imaging for another four days. Linear track. Mice were trained to run on the linear track for four days during the training session. The one-meter linear track is made of black plastic materials. Two water reward zones were located at the end of the linear track. The walls of reward zones were adorned with various visual cues as spatial information. $10 \%$ ethanol was used to get rid of the odor cues left from other mouse in the previous behavioral session. In the beginning of each session, a mouse was released in the middle of the track and ran to the end of track to get 10ul of water reward. Once the mouse reached the minimal 50 laps within 10 minutes in a training session, we start experimental recording in the next day. On the first day of the miniscope recording, animals were required to complete two sessions on the same track with a horizontal direction. Each mouse had a two-minute break time in the home cage during each session. At least 40 laps per session were acquired for the data processing. On day 2, the mouse ran on the same linear track with a 
horizontal direction as day 1 in session 1 . Then, the linear track was rotated in a 90 -degree direction, named as "vertical" relative to the recording environment. After 2 minutes of rest in the home case, the animal went through another 40 laps on the vertical track. On the third day, the animal ran on the vertical track in session 1 then back to the horizontal track in session 2 .

\section{Data preprocessing}

The raw calcium recordings were down-sampled to 15 frames/sec. Motion correction was applied to the neuron recordings with the well-established motion correction streamline NormCorre (Pnevmatikakis and Giovannucci, 2017), to fix the rigid movements caused by potential scope movement across time.

Extract neural calcium signal using CNMF-E method. The calcium signal of each neuron was gathered from video data using the Extended Constrained Nonnegative Matrix Factorization (CNMF-E) framework proposed by Zhou et al (Zhou et al., 2018b). This framework uses the following model to represent the video data:

$$
y(x, t)=\sum_{i=1}^{K} a_{i}(x) * c_{i}(t)+b(x, t)
$$

Where $y(x, t)$ represents the raw video data, $a_{i}(x)$ represents the neuron's spatial footprint, $c_{i}(t)$ represents the calcium activity and $b(x, t)$ represents the background activity. By applying sophisticated background approximation, CNMF-E can efficiently remove baseline noise, and achieve the neuron footprint and calcium response via deconvolution. To remove false positive detections, a 2D Gaussian kernel was fit for the spatial footprint of each neuron, and KullbackLeibler divergence was calculated between the footprint and Gaussian kernel. Kullback-Leibler divergence quantifies the similarity between two distributions, and here it represents the closeness between the actual neuron footprint and the theoretically perfect footprint. Neurons with divergence values larger than 0.6 were kept for subsequent analyses. 
CNMF-E was applied to different trials independently. To identify common neurons across the trials, for each pair of extracted neurons across two trials, we calculated the level of footprint overlap between the two neurons, and the similarity of distance pattern between each neuron and its neighbors. Both measures were converted to probability values indicating the chance for the two neurons to be aligned, and the final alignment probability was the average of the overlap probability and distance pattern probability. The neuron pairs with the highest alignment probability go beyond 0.25 was considered as the same neuron across two trials.

Mouse movement behavior extraction. The movement trajectory was extracted from the behavioral tracking videos simultaneously recorded with neuronal responses, at a sample rate of $30 \mathrm{~Hz}$. The bottom of the box was selected as the region of interest (ROI), to restrict the area for behavioral detection. The trajectory was defined as the positions of red LED centroid across all frames and was smoothed using moving average method.

Spatial rate map calculation. The calcium spike trains were calculated by applying the CNMFE embedded deconvolution algorithm, OASIS (Friedrich et al., 2017), to the extracted temporal calcium dynamics. A threshold of $10 \%$ of maximum amplitude of the neurons' spike train was set for each neuron, and potential events with an amplitude lower than the threshold were excluded.

The movement trajectories were aligned with the calcium responses according to the timestamp that record the correspondence between behavioral recordings and calcium recordings. The ROI was divided into $10 \mathrm{~mm}$ X10 mm bins. The total time mouse spent inside a bin was counted as bin time (sec). The total number of calcium peaks above threshold was added up and normalized by bin time to achieve event rate. When presenting, smoothing was applied to the event rate map with a $100 \mathrm{~mm}$ X100 mm 2D Gaussian kernel (delta $=20 \mathrm{~mm}$ )

dF/F calculation. When comparing amplitude values, the neuronal response of each neuron was normalized to $\mathrm{dF} / \mathrm{F}$, which was calculated as $\frac{\mathrm{dF}}{\mathrm{F}}=\frac{d F-\text { mean }(d F)}{\text { mean }(d F)}$ 
Information score and place cell. Information score of recorded neurons was calculated as information per second and information per spike introduced by Skaggs et al. in 1993. Only the running sessions with speed larger than $0.5 \mathrm{~cm} / \mathrm{s}$ were included in calculation, and the spatial bins with bin time smaller than $0.1 \mathrm{sec}$ were excluded to avoid non-existed trespass caused by trajectory smoothing. For a rate map with $\mathrm{n}$ bins, the information per second is defined as:

$$
\begin{aligned}
& \text { Information Score (bits/second) }=\sum_{i=1}^{n} P_{i} \lambda_{i} \log _{2} \frac{\lambda_{i}}{\lambda} \\
& \text { Information Score (bits/spike) }=\sum_{i=1}^{n} P_{i} \frac{\lambda_{i}}{\lambda} \log _{2} \frac{\lambda_{i}}{\lambda}
\end{aligned}
$$

$P_{i}$ is the probability the mouse stays in the $i$ th bin, which is represented as the ratio between the times in the bin and total times of the trial. $\lambda_{i}$ is the firing rate of the $i$ th bin, while $\lambda$ is the average firing rate across the trial. Place cell was defined by comparing the information per spike of each neuron with its shuffled baseline. The calcium responses were divided into 100 trunks and randomly shuffled for 100 times, to disrupt the correspondence with behaviors and generate a distribution of potential score values the neuron may achieve. A neuron was determined as a place cell if its original score value was higher than the 95th percentile of the shuffled distribution.

Sparsity. Sparsity is a measure of the fraction of environment in which the cell is active (Skaggs et al., 1996). The definition is Sparsity $=\frac{\Sigma\left(p_{i} \lambda_{i}\right)^{2}}{\Sigma p_{i} \lambda_{i}{ }^{2}}$, where $p_{i}$ is the probability the mouse stays in the $i$ th bin, which is represented as the ratio between the times in the bin and total times of the trial, and $\lambda_{i}$ is the firing rate of the $i$ th bin.

Spatial coherence. Spatial coherence measures the level of spatially contiguous activity the neuron exhibits, that is if the spatial bins with high activity is located close to each other, a high Spatial coherence score would be achieved (Zhang et al. 2014). Spatial coherence was 
calculated as the Pearson correlation between the activity of each spatial bin and the average activity of its $3 \times 3$ neighboring bins.

Statistical analyses. Data are presented as the mean \pm s.e.m, unless otherwise specified. Data analysis was conducted using MATLAB and R. Because the data presented in this article were collected using different experimental designs, several appropriate statistical tests were applied in the data analysis. This included the ANOVA, linear mixed-effect model (LME), and Kolmogorov-Smirnov test. The main idea of LME ("fitlme" in MATLAB) is to take the inherent correlations in correlated data, such as the neurons from the same mouse, into consideration when conducting statistical modeling and hypothesis testing (Laird and Ware, 1982; Mclean et al., 1991). For example, regarding the comparison between Non-Tg young and AD young mouse cells, measurements of 1180 cells from 5 Non-Tg young mice and 1076 cells from 8 AD young mice are presented in Figure 3A. We fitted an LME by using genotype for a fixed effect and mouse id for a random effect. The importance of LME and its more generalized versions has been increasingly recognized in recent studies involving large cell sample data collected from a relatively small number of animals (Indersmitten et al., 2019; Stobart et al., 2018). For cumulative distributions, the Kolmogorov-Smirnov (KS) test was applied for analysis of the difference. The level of statistical significance was defined as $p \leq 0.05$.

\section{Results}

\section{Age-dependent amyloid- $\beta$ and tau pathology in 3xTg-AD mice}

We used triple-transgenic Alzheimer's disease (3xTg-AD) model mice that overexpress

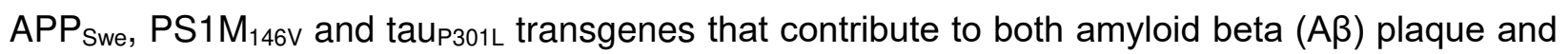
neurofibrillary tangle formation (Belfiore et al., 2019; Oddo et al., 2003). Unlike most other mouse models, 3xTg-AD model mice uniquely develop two AD hallmark pathological features: plaque and tangle formation (Mesulam, 2000; Oddo et al., 2003). To determine age-related and AD progression effects, we compared littermate control and AD-like mice at young and old ages 
(young age, 3.6 - 6.5 months old versus old age, 18 - 21 months old). The age choices are guided by behavioral and neuropathological findings by our and other groups (Baglietto-Vargas et al., 2018; Belfiore et al., 2019; Mesulam, 2000; Oddo et al., 2003; Stimmell et al., 2019).

To measure age-dependent plaque and tau aggregate formation in the $A D$ mouse hippocampus, we immunostained 3xTg-AD and control genetic background non-transgenic (Non$\mathrm{Tg}$ ) sections with $A \beta$ and Tau antibodies. All hippocampal sample sections were from mice that had undergone imaging and behavioral experiments (see below), thus these results can be correlated with AD neuropathology. Across at least 3 different sets of immunostaining experiments, we confirm that 6.5-month-old $3 \times \mathrm{Tg}-\mathrm{AD}$ mice exhibit intracellular $A \beta$ accumulation in hippocampal pyramidal neurons visualized by immunostaining with the $6 \mathrm{E} 10$ antibody shown in red, counterstained with DAPI in blue showing cell nuclei (Fig. 1A, bottom left). Extracellular $A \beta$ deposits are distributed throughout the CA1 pyramidal layers and extend to all layers of the hippocampus in 3xTg-AD mice by 18 months (Fig. $1 A$, bottom right). In contrast, no $A \beta$ deposits are detected in the control Non-Tg mice at either young (Fig. 1A, top left, 6.5 months) or old ages (18 months) (Fig. 1A, top right). The phospho-tau (Ser202, Thr205) monoclonal antibody (AT8) staining, shown in red and counterstained with DAPI shown in blue, was used to identify the presence of human tau pathology in 3 different sets of immunostaining experiments. Anti-AT8 staining shows that tau aggregates in a number of CA1 pyramidal neurons in aged 3xTg-AD mice (18-month-old, white arrow, Fig. 1B, bottom right). In comparison, only sparse AT8+ cells are found in hippocampal CA1 in younger 3xTg-AD mice aged 6.5-months (Fig. 1B, bottom left). Tau pathology is not detected in the young and old control Non-Tg mice (Fig. 1B, top left and right). Consistent with previous work, $A \beta$ accumulation is detectable at an earlier age than tau pathological accumulation in 3xTg-AD mice, and both neuropathological features are absent in age matched control Non-Tg mice (Belfiore et al., 2019; Oddo et al., 2003). While age-dependent pathological changes correlate with AD-related memory behavioral deficits (Belfiore et al., 2019; 
Stimmell et al., 2019), spatial coding of in vivo neural circuit activities in the AD mouse model has not been studied during exploration of environments.

\section{Miniscope imaging of neural population activities in 3xTg-AD hippocampal CA1}

To determine whether hippocampal CA1 ensemble activities during spatial exploration are altered in an age-dependent fashion in 3xTg-AD mice, we used a head-mounted miniscope to image in vivo calcium transient ensemble activities in the 3xTg-AD mouse hippocampal CA1, and compared ensemble activities with age-matched control Non-Tg animals sharing the same genetic background (Fig. 2). To visualize neural calcium activity in the dorsal hippocampal CA1 region, mice were injected with the AAV1-CamKII(0.4)-GCaMP6f virus to restrict expression to CA1 excitatory neurons (Fig. 2A). This $0.4 \mathrm{~Kb}$ promoter is derived from murine $\alpha$ Calcium/calmodulin-dependent kinase II (CaMKII), and is reported to show up to $95 \%$ specificity to excitatory neurons in the cerebral cortex (Scheyltjens et al., 2015).

The cortex overlying the hippocampus was removed, then a gradient refractive index GRIN lens was implanted above CA1 (Fig. 2B). The implanted GRIN lens allows repeated and longitudinal imaging of the same group of neurons in single cell resolution across prolonged periods up to weeks and months. A small metal baseplate is glued around the lens, and the headmounted miniscope is magnetically attached to the baseplate. As established in our published studies (Grieco et al., 2021; Grieco et al., 2020; Sun et al., 2019; Wong et al., 2021), GRIN lens implants and the stability of the miniscope attachment permit longitudinal dynamic imaging results to be aligned across sessions and multiple days due to stable registration. Hundreds of neurons can be captured simultaneously in a $700 \mu \mathrm{m} \times 450 \mu \mathrm{m}$ field of view, with a resolution of $\sim 0.9 \mu \mathrm{m}$ per pixel (Fig. 2C).

Spatial footprints of individual neurons and their corresponding calcium transients were detected and extracted from raw video data using the established CNMF-E method (Zhou et al., 2018a) (Fig. 2C-D). Overall we find that the extracted neuronal numbers in AD mice are less than 
control mice per the field of view (Fig. 2C), as would be expected due to neurodegenerative loss. By averaging the numbers of extracted neurons for combined age groups across open field exploration trials, 3xTg-AD hippocampal CA1 imaging fields show $331 \pm 41$ (mean \pm s.e.m) cells while Non-Tg mice hippocampal CA1 imaging fields show $456 \pm 50$ cells (two-way ANOVA, $p=$ $0.0062, F(1,28)=8.774)$. The aged group overall has a lower number of extracted neurons than the young group (538 \pm 48 cells in the young group versus $281 \pm 29$ cells in the aged group; twoway ANOVA, $p=0.001, F(1,28)=24.97)$. This suggests that fewer cells per unit of space may be active in $A D$ and aging conditions.

To quantitatively characterize hippocampal neuronal ensemble activities during free exploration, we constructed spatial firing rate maps for the extracted CA1 neurons during animal exploration in open circle (diameter, $36 \mathrm{~cm}$ ) and square $(26 \mathrm{~cm} \times 26 \mathrm{~cm})$ arenas. Arenas were divided into $1 \mathrm{~cm} \times 1 \mathrm{~cm}$ spatial bins. The binned calcium transient rate is defined by dividing the total number of calcium activity peaks by the total exploration times within each bin. Transients as defined by sharp peaks in activity exceeding $10 \%$ of the maximum peak amplitude. The spatial firing rate maps of example neurons of both ages and genotypes are shown in Fig. 2E-H. For Non-Tg mice at both ages, individual excitatory CA1 neurons are able to display calcium firing activities restricted to specific locations, which is consistent with the well-described 'place-specific' action potential firing of CA1 excitatory neurons (Fig. 2E, G). For 3xTg-AD mice at both ages, individual excitatory CA1 neurons show less place-specific activities as characterized by more diffused firing and less in-field event rates (Fig. 2F, H), which suggests decreased signal-to-noise ratio and potential impairment of spatial representations in CA1.

\section{Age- and AD- dependent neural activity differences in mouse hippocampal CA1 during open field exploration}

To further quantitatively investigate the $\mathrm{AD}$ - and age-related differences in hippocampal excitatory CA1 neurons activities, we compared the calcium transient rates and calcium event 
amplitudes of hippocampal CA1 neurons from 3xTg-AD and Non-Tg genotypes at different ages (Fig. 2D, Fig. 3A and B). For the young age group, overall the 3xTg-AD genotype displays a significantly higher calcium event rate compared with the Non-Tg control (Fig. 2E-F, Fig. 3A. NonTg young: $0.0752 \pm 0.0010 \mathrm{~Hz}$ (1180 cells from 5 mice); AD young: $0.0965 \pm 0.0013 \mathrm{~Hz}(1076$ cells from 8 mice); linear mixed-effects $(L M E)$ model analysis: $p=0.0091)$. Similarly, for the old age group, on average the old AD mice exhibit a higher firing rate than old Non-Tg mice (Fig. 2GH, Fig. 3A. Non-Tg old: $0.0497 \pm 0.0012 \mathrm{~Hz}$ (716 cells from 9 mice); $3 x T$ T-AD old: $0.0612 \pm 0.0018$ $\mathrm{Hz}$ (430 cells from 8 mice); LME: $\mathrm{p}=0.0094)$. We also note that for both control and $3 x \operatorname{Tg}-\mathrm{AD}$ mice, age decreases the overall rate of excitatory neural calcium activities in mouse CA1 (NonTg young vs Non-Tg old, LME: $p=3.8744 \times 10^{-5} ; 3 x \operatorname{Tg}-A D$ young vs 3xTg-AD old, LME: $p=1.5095$ $\left.\times 10^{-5}\right)$.

In terms of the overall calcium event amplitudes $(\Delta F / F)$, we find that $3 x T g-A D$ genotype exhibits lower amplitudes than Non-Tg mice at the young age (Fig. 2E-F, Fig. 3B. Non-Tg young: $7.386 \pm 0.100$ in the unit of $d F / F$ (1180 cells from 5 mice); $3 x T g-A D$ young: $5.725 \pm 0.064 \mathrm{dF} / F$, (1076 cells from 8 mice), LME: $p=0.0066$ ). The calcium amplitudes are generally higher for the

old age group, compared with the young age group (Fig. 2G-H, Fig. 3B. Non-Tg old: $11.680 \pm$ $0.322 \mathrm{dF} / \mathrm{F}$ (716 cells from 9 mice); $3 x T g-A D$ old: $11.700 \pm 0.619 \mathrm{dF} / \mathrm{F}$ (430 cells from 8 mice). Non-Tg young vs Non-Tg old: LME: $p=0.0055$, 3xTg-AD young vs 3xTg-AD old: LME: $p=0.0287$ ). The 3xTg-AD and Non-Tg genotypes did not differ significantly at the old age (LME: $p=0.6388$ ).

These data suggest that 3xTg-AD mouse hippocampal circuits are associated with higher neural ensemble activities relative to Non-Tg controls at either age, which is supported by neural circuit hyper-activity with the accumulation of $A \beta$ as observed in anaesthetized $A D$ mouse preparations (Busche et al., 2012; De Strooper and Karran, 2016; Harris et al., 2020).

\section{Spatial coding impairments of 3xTg-AD CA1 neurons during open field exploration}


Given that 3xTg-AD mice show hippocampal spatial memory impairments (BagliettoVargas et al., 2018; Oddo et al., 2003; Stimmell et al., 2019), we next investigated the differences of CA1 neuron spatial coding abilities between 3xTg-AD and Non-Tg genotypes at different ages. Using tetrode electrical recordings, a previous study using a different AD mouse model (single APP knock-in, APP-KI) shows that the APP-KI mice exhibit deteriorated spatial tuning represented by lower information score values (Jun et al., 2020). We used a similar approach by calculating the information scores in bits/spike (bits/event) from pooled CA1 place cells of each genotype and age group, and comparing their cumulative distributions. The metrics of bits/spike and bits/second measure different aspects of activity across sub-regions (bins) of an environment. Both the bits/second and bits/spike metrics utilize the distributions of firing rate across binned locations in an environment (Skaggs et al., 1999). The bits/spike metric is increased when a subset of positional firing rates is high against a low mean positional firing rate. Because of this, neurons with place specific activity tend to have high bits/spike values.

We find spatial coding impairments of 3xTg-AD hippocampal CA1 neurons in freely moving animals during open field exploration. The bits/event metric shows that neural calcium events of the 3xTg-AD mice encode less information than Non-Tg mice, at either the young or old age (Fig. 3C; the respective 50\%-cumulative values for Non-Tg young (233 place cells from 5 mice), AD young (214 place cells from 8 mice), Non-Tg old (104 place cells from 9 mice) and 3xTg-AD old (131 place cells from 8 mice) are 3.4681, 3.0372, 4.2743 and 3.4172. Non-Tg young vs 3xTg-AD young distributions: $p=4.1683 \times 10^{-12}$. Non-Tg old vs $3 \times T g-A D$ old: $p=5.2701 \times 10^{-}$ 7, Non-Tg young vs Non-Tg old: $p=6.3021 \times 10^{-12}, 3 \times T g-A D$ young vs 3xTg-AD old: $p=1.1370 \times$ 10-6, two-sample Kolmogorov-Smirnov test).

To further compare the spatial coding abilities of hippocampal CA1 excitatory neurons across the genotype and age groups, we measured the sparsity and spatial coherence (Jung et al., 1994; Zhang et al., 2014) of calcium event rate maps for all CA1 neurons pooled from each mouse group. The sparsity index measures the fraction of the exploration area where a neuron 
fires spikes, and lower sparsity indicates more constrained firing activities at specific locations (Jung et al., 1994). Coherence measures the extent to which adjacent positional firing rate bins share similar firing rates. For truly place-specific activity, coherence is high because spatial bins with high rates are concentrated in one location. We find that at either age, 3xTg-AD CA1 cells exhibit higher sparsity values than Non-Tg, which complements the bits/event measures and indicate the lower spatial selectivity of place cell firing in 3xTg-AD mice [Fig. $3 \mathrm{E}$; the respective $50 \%$-cumulative values for Non- $\mathrm{Tg}$ young (1180 cells from 5 mice), 3xTg-AD young (1076 cells from 8 mice), Non-Tg old (716 cells from 9 mice) and 3xTg-AD old (430 cells from 8 mice) are $0.0746,0.0957,0.0492$ and 0.0657 . Non-Tg young vs $3 \times T g-A D$ young: $p=1.2703 \times 10^{-31}$, NonTg old vs 3xTg-AD old: $p=2.0458 \times 10^{-7}$, two-sample Kolmogorov-Smirnov test). Further, for both 3xTg-AD and Non-Tg, mice in the young age group have a higher sparsity than the old age group (Fig. 3 E; Non-Tg young vs Non-Tg old: $p=9.0277 \times 10^{-42} ; 3 \times T g-A D$ young vs $3 \times T g-A D$ old: $p=$ $1.0343 \times 10^{-25}$, two-sample Kolmogorov-Smirnov test). In addition, the 3xTg-AD mice show overall higher coherence values of activity rate maps than Non-Tg mice at the old age (Fig. $3 \mathrm{~F}$; the respective $50 \%$-cumulative- values for Non-Tg young (1180 cells from 5 mice), 3xTg-AD young (1076 cells from 8 mice), Non-Tg old ( 716 cells from 9 mice), and 3xTg-AD old (430 cells from 8 mice): $0.4369,0.4437,0.4123$ and 0.4286 . Non-Tg young vs $3 x T g-A D$ young: $p=0.0728$, Non-Tg old vs $3 \times T$ Tg-AD old: $p=5.4990 \times 10^{-4}$, two-sample Kolmogorov-Smirnov test). Younger mice show higher coherence than older mice for both genotypes (Fig. 3 F; Non-Tg young vs NonTg old: $p=2.3031 \times 10^{-6}, 3 \times T g-A D$ young vs $3 \times T g-A D$ old: $p=0.0177$, two-sample KolmogorovSmirnov test). As spatial coherence measures the correlation between the activity of each bin and the averaged activities of its neighborhood bins in a rate map (Jung et al., 1994), we reason that the more diffuse firing fields of old 3xTg-AD mice may result in higher spatial coherence.

\section{Spatial coding deficits of 3xTg-AD CA1 neurons in linear track trials}


We extended our investigation to a linear track environment. Population calcium transient activities in hippocampal CA1 were imaged with head-mounted miniscopes while mice ran along a 1-meter linear track (width, $3 \mathrm{~cm}$ ) for a water reward at each end. CA1 excitatory neurons that exhibit place selective activities can be identified from the populations across the genotypes and ages (Fig. 4 A-D). We quantitatively analyzed the place-specific activities in linear track trials, as we did for open field exploration. We find that the calcium event rates across mouse groups do not exhibit significant differences (Fig. 4E), but AD mice show a significant difference from NonTg mice in terms of calcium event amplitudes [Fig. 4 F; Non-Tg young (1742 cells from 6 mice): $5.745 \pm 0.112 \mathrm{dF} / \mathrm{F} ; 3 x T g-A D$ young (1988 cells from 8 mice): $4.534 \pm 0.076 \mathrm{dF} / \mathrm{F} ;$ Non-Tg old (1136 cells from 5 mice): $4.573 \pm 0.107 \mathrm{dF} / \mathrm{F} ; 3 x \operatorname{Tg}-\mathrm{AD}$ old (1560 cells from 5 mice): $5.064 \pm 0.079$ dF/F. Non-Tg young vs 3xTg-AD young, LME: $p=0.0167$; Non-Tg old vs 3xTg-AD old, LME: $p=0.0495 ;$ Non-Tg young vs Non-Tg old, LME: $p=0.0132 ; 3 x T g-A D$ young vs $3 x T g-A D$ old, LME: $p=0.0354)$.

Consistent with our finding in mouse exploration of open fields, we find that for both ages, 3xTg-AD mice exhibit lower spatial information score in bits/event than Non-Tg mice [Fig. 4G; the respective $50 \%$-cumulative values for Non-Tg young (238 place cells in direction $1+246$ place cells in direction 2 , from 6 mice), 3xTg-AD young (213 place cells in direction $1+237$ place cells in direction 2, from 8 mice), Non-Tg old (163 place cells in direction $1+159$ place cells in direction 2, from 5 mice) and 3xTg-AD old are (259 place cells in direction $1+272$ place cells in direction 2, from 5 mice) are $2.8194,2.6591,2.2647$ and 1.9509. Non-Tg young vs 3xTg-AD young: $p=0.0432$, Non-Tg old vs 3xTg-AD old: $p=0.0010$, Non-Tg young vs Non-Tg old: $p=8.7241 \times 10^{-}$ 7, 3xTg-AD young vs 3xTg-AD old: $p=3.4423 \times 10^{-5}$, two-sample Kolmogorov-Smirnov test). During linear track trails, hippocampal CA1 excitatory neurons in old 3xTg-AD mice have higher sparsity values than young 3xTg-AD mice, while for control mice, there are no significant differences between ages [Fig. 4H; the respective 50\%-cumulative values for Non-Tg young (1742 cells (each direction) from 6 mice), 3xTg-AD young (1988 cells (each direction) from 8 
mice), Non-Tg old (1136 cells (each direction) from 5 mice) and 3xTg-AD old (1560 cells (each direction) from 6 mice) are $0.0490,0.0429,0.0499$, and 0.0559 . Non-Tg young vs 3xTg-AD young: $p=1.2231 \times 10^{-9}$, Non-Tg old vs 3xTg-AD old: $p=1.7939 \times 10^{-5}$, Non-Tg young vs Non-Tg old: $p$ $=0.0818 ; 3 \times T g-A D$ young vs $3 \times T g-A D$ old: $p=3.4707 \times 10^{-31}$, two-sample Kolmogorov-Smirnov test). 3xTg-AD mouse cells exhibit higher spatial coherence values than Non-Tg mice [Fig. 4l; the respective 50\%-cumulative values for Non-Tg young (1742 cells (each direction) from 6 mice), 3xTg-AD young (1988 cells (each direction) from 8 mice), Non-Tg old (1136 cells (each direction) from 5 mice) and , 3xTg-AD old (1560 cells (each direction) from 6 mice) are $0.3573,0.3658$, 0.3632 and 0.3778 . Non-Tg young vs 3xTg-AD young: $p=8.8051 \times 10^{-10}$, Non-Tg old vs $3 \times T g$ $A D$ old: $p=2.7640 \times 10^{-11}$, Non-Tg young vs Non-Tg old: $p=7.9276 \times 10^{-12}, 3 \times T g-A D$ young vs $3 \times T g-$ $A D$ old: $p=2.7437^{*} 10^{-17}$, two-sample Kolmogorov-Smirnov test). Thus compared with control mice, $C A 1$ excitatory neurons in 3xTg-AD mice show spatial coding deficits in the linear track environment.

\section{Locomotion modulation of 3xTg-AD mouse CA1 neural population activities}

It is known that locomotion states have significant effects across rodent sensory systems including visual and auditory circuits (Arriaga and Han, 2017a). While both the calcium activities of CA1 excitatory neurons and inhibitory neurons can be modulated by locomotion (Fuhrmann et al., 2015; Góis and Tort, 2018), locomotion effects on hippocampal neural calcium population activities have not yet been studied in freely moving animals. Miniscope imaging has an advantage of monitoring neural ensemble activities in hippocampal CA1 of animals during free moving conditions, thus providing the opportunity to examine the modulatory effects of free locomotion on CA1 neural calcium activity ensembles across different groups.

As shown in Fig. 5A, the calcium activities of hundreds of excitatory neurons recorded during one session from each example mouse are displayed in raster plots over the exploration time. We delineate the high-velocity epochs (black vertical bars) below the raster plots to enable 
visual comparisons between the neural activity level and locomotion velocity. The high-velocity epoch is defined as the duration when the velocity goes above the averaged trial velocities of each group (Fig. 5B). To further illustrate the relationship between population calcium responses and velocity overtime, we align the z-scored ensemble calcium response trace with the $z$-scored velocity trace. Interestingly, compared with control mice at the young or old age, overall neural population calcium activities in hippocampal $C A 1$ of $A D$ mice appear to be more closely modulated by locomotion velocities and CA1 ensemble amplitudes are positively correlated with locomotion velocity changes (Fig. 5A).

We quantitatively examined the relationship between calcium activity amplitude and velocity by calculating the correlation between individual neurons' calcium responses and velocities. We apply a $15 \mathrm{sec}$ Gaussian window (2.5 sec standard deviation) to smooth both calcium response and movement velocity traces in the time-series, then compare the cumulative distributions of correlations across different genotypes and ages. Our quantification confirms our qualitative observations in Fig. 5A. Compared with age-matched controls, young and old 3xTgAD CA1 neurons show stronger correlations between calcium response amplitudes and locomotion velocities [Fig. 5C; the respective 50\%-cumulative values for Non-Tg young (1180 cells from 5 mice), 3xTg-AD young (1076 cells from 8 mice), Non-Tg old ( 716 cells from 9 mice) and 3xTg-AD old (430 cells from 8 mice) are $0.0435,0.0561,0.0341$ and 0.093 . Non- $T g$ young vs 3xTg-AD young: $p=9.2871 \times 10^{-6}$, Non-Tg old vs $3 \times T g-A D$ old: $p=1.6663 \times 10^{-10}$, two-sample Kolmogorov-Smirnov test). This holds true when we only include place cells for analysis. Place cells were defined as cells with spatial information score above the chance level (see details in the Method). The locomotion effects appear to be more robust for the place cells [Fig. 5D; the respective $50 \%$-cumulative values for Non-Tg young (233 place cells from 5 mice), $3 \times \mathrm{Tg}-\mathrm{AD}$ young (214 place cells from 5 mice), Non-Tg old (104 place cells from 9 mice) and 3xTg-AD old (131 place cells from 5 mice) are $0.1228,0.1560,0.0901$ and 0.1556 . Non- $T g$ young vs $3 x T g-A D$ young: $p=0.0093$, Non-Tg old vs $3 x T g-A D$ old: $p=0.0016$, two-sample Kolmogorov-Smirnov test). 


\section{Discussion}

Previous work on AD has focused on molecular and neuropathological features as they correlate to cognitive defects. AD research is now increasingly focused on mechanistic changes at the level of neuronal circuits (Harris et al., 2020). In the present study, we have examined neural calcium activities of hippocampal CA1 neuronal populations in freely behaving 3xTg-AD mice and controls at young (3 - 6.5 months on average) and old (18 - 21 months old) ages. We leveraged the advantages of miniscope-based GCaMP calcium imaging to examine the impairments of spatial representation and spatial coding across large neuronal ensembles by comparing hippocampal CA1 excitatory neural ensemble activities during open-field exploration, and track-based route-running behaviors in age-matched $A D$ and control mice. We have identified AD- and age-related differences in neural calcium activities, and have determined deficits in spatial information coding in hippocampal neural ensembles in the 3xTg-AD mouse model. We also find locomotion significantly modulates the amplitude of hippocampal neural ensemble activities in 3xTg-AD mice, but not in non-transgenic controls during open field exploration.

Many transgenic AD models have been used in the research studies to date, but most models only contain mutation genes for the progression of $A \beta$ plaques, such as APP Tg2576, APP/PS1 and 5xFAD. These models are convenient for the detection of $A \beta$ accumulation at early age but do not take into account the tau pathology in the late AD stage. In our study, we used the 3xTg-AD mouse model that contains the genetic mutations contributing to both pathological markers (Mesulam, 2000; Oddo et al., 2003). AD related neurodegeneration may affect hippocampal CA1 neural activation as 3xTg-AD hippocampal CA1 imaging fields have a smaller number of extracted neurons compared to control mouse hippocampal CA1 fields. Aging might also have effects, as the aged group also has a lower number of extracted neurons relative to the young group. The age-dependent plaque and tau aggregate formation in the $3 \times \mathrm{Tg}$ - 
AD mouse hippocampus is correlated well with CA1 neural activity alterations identified in our imaging experiments across the ages and genotypes. Our imaging results are also supported by the deficiency in spatial memory behaviors observed in 3xTg-AD mice (Baglietto-Vargas et al., 2018; Oddo et al., 2003; Stimmell et al., 2019).

We identify $A D$ - and age- dependent neural activity differences in mouse hippocampal CA1 during open field exploration. We find that 3xTg-AD CA1 excitatory cells have significantly higher calcium firing rates compared with controls at both young or old ages, indicating that in vivo enhanced neuronal ensemble activity is a disease feature. Increased ages are correlated with decreased neural calcium firing rates across genotypes. We also have examined calcium event amplitudes. We find that 3xTg-AD CA1 cells have lower amplitudes than control mice at the young age, and that neural calcium amplitudes are generally higher for AD and control CA1 cells, compared with the cells of young age groups. These data suggest AD and aging share related alterations in neural activities and calcium homeostasis. The higher neural ensemble calcium activities found in 3xtg AD mouse hippocampal CA1 may be a critical AD disease feature. which is supported by neural circuit hyper-activity observed in anaesthetized AD mouse preparations (Busche et al., 2012; De Strooper and Karran, 2016; Harris et al., 2020).

Our data demonstrate spatial coding defects of hippocampal CA1 ensemble activities in 3xTg-AD mice, as our overall imaging results in both open field and linear track environments indicate that neuronal populations of $3 \times T g-A D$ mice show lower spatial information scores compared with control mice. Spatial firing of CA1 neurons of old 3xTg-AD mice also displays higher sparsity and spatial coherence compared with those from control mice. This supports the notion that place-specific firing fields of CA1 neurons in the AD condition tend to be larger in size, which indicates less place specificity for spatial representation. 
Our finding that locomotion significantly modulates the amplitude of hippocampal neural ensemble activities in 3xTg-AD mice, but not in non-transgenic controls is another important new finding. During movement, hippocampal local field potential (LFP) activity is characterized by $\theta$ frequency oscillations; in contrast, during awake immobility, LFP activity is punctuated by large, irregular activity containing periods of sharp-wave/ripple (SWR) events. Much current research related to this is focused on hippocampal interneurons as their activity is highly modulated with locomotion speed (Arriaga and Han, 2017b; Góis and Tort, 2018), while locomotion modulates excitatory neurons to a lesser degree (Góis and Tort, 2018). Given that AD neurodegeneration can alter circuit excitation/inhibition balance, it can be inferred that the speed-correlated inhibition of pyramidal neurons will be weakened in the AD condition. Indeed, our results indicate that CA1 place cells from 3xTg-AD mice exhibit obviously stronger speed modulation on their neural calcium activity amplitudes. Whether decrease in inhibition will lead to higher activity modulation of excitatory neurons in $A D$ related neural circuits requires further investigation.

\section{Conclusions}

In conclusion, we applied miniscope calcium imaging in 3xTg-AD model and control mice to determine the AD- and aging- related impacts on hippocampal neural population activities. Our data show neural activity alterations and spatial coding defects in CA1 neuronal ensembles that correlate with the development of $A D$ pathology and AD-related spatial memory behavioral deficits.

\section{Abbreviations}

3xTg-AD : Triple-transgenic Alzheimer's disease mouse model

5xFAD: five familial Alzheimer's disease (FAD) mutations 
AAV: Adeno-associated virus

AD: Alzheimer's disease

AP: Anteroposterior

APP-KI: Amyloid precursor protein-knock in mouse model

$A \beta$ : amyloid beta

CaMKII: Calcium/calmodulin-dependent kinase II

CNMF-E: Extended Constrained Nonnegative Matrix Factorization

DV: Dorsoventral

GRIN: Gradient refractive index

KS: Kolmogorov-Smirnov

LME: linear mixed-effect model

Miniscopes: Miniature fluorescent microscopes

ML: Mediolateral

Non-Tg: Non-transgenic mouse model

OASIS: Online Active Set method to Infer Spikes

PBS: Phosphate buffered saline

ROI: Region of interest

SUB: Subiculum

\section{Declarations}

\section{Ethics approval and consent to participate}

All experiments were conducted according to the National Institutes of Health guidelines for animal care and use and were approved by the Institutional Animal Care and Use Committee and the Institutional Biosafety Committee of the University of California, Irvine.

\section{Consent for publication}


All authors have approved the final manuscript and agree with its submission to Molecular Neurodegeneration.

\section{$\underline{\text { Availability of data and materials }}$}

The datasets used for the present study are available from the corresponding author upon reasonable request.

\section{Competing interests}

The authors declare no competing interests.

Funding

This work was supported by US National Institutes of Health (NIH) grants (R01NS078434, R01NS104897 and RF1AG065675) to X.X. and D.N., R35 GM127102 to T.C.H..

\section{$\underline{\text { Author contributions }}$}

X.L. and Q.Y. performed imaging, behavioral and immunostaining experiments. L.J., X.L., D.B., X.X., T.C.H., D.N. analyzed the data, prepared the figures and wrote the manuscript with the help from other authors. X.X. designed and oversaw the project. All authors read and approved the final manuscript.

\section{Acknowledgments}

This work was supported by US National Institutes of Health (NIH) grants (R01NS078434, R01NS104897 and RF1AG065675) to X.X. and D.N., R35 GM127102 to T.C.H.. 


\section{References}

Alzheimer's Association (2020). 2020 Alzheimer's disease facts and figures. Alzheimer's \& dementia : the journal of the Alzheimer's Association.

Arriaga, M., and Han, E.B. (2017a). Dedicated Hippocampal Inhibitory Networks for Locomotion and Immobility. J Neurosci 37, 9222-9238.

Arriaga, M., and Han, E.B. (2017b). Dedicated Hippocampal Inhibitory Networks for Locomotion and Immobility. The Journal of Neuroscience 37, 9222.

Baglietto-Vargas, D., Prieto, G.A., Limon, A., Forner, S., Rodriguez-Ortiz, C.J., Ikemura, K., Ager, R.R., Medeiros, R., Trujillo-Estrada, L., Martini, A.C., et al. (2018). Impaired AMPA signaling and cytoskeletal alterations induce early synaptic dysfunction in a mouse model of Alzheimer's disease. Aging cell 17, e12791.

Bast, T., Pezze, M., and McGarrity, S. (2017). Cognitive deficits caused by prefrontal cortical and hippocampal neural disinhibition. Br J Pharmacol 174, 3211-3225.

Belfiore, R., Rodin, A., Ferreira, E., Velazquez, R., Branca, C., Caccamo, A., and Oddo, S. (2019). Temporal and regional progression of Alzheimer's disease-like pathology in 3xTgAD mice. Aging cell 18, e12873.

Billings, L.M., Oddo, S., Green, K.N., McGaugh, J.L., and LaFerla, F.M. (2005). Intraneuronal Abeta causes the onset of early Alzheimer's disease-related cognitive deficits in transgenic mice. Neuron 45, 675-688.

Busche, M.A., Chen, X., Henning, H.A., Reichwald, J., Staufenbiel, M., Sakmann, B., and Konnerth, A. (2012). Critical role of soluble amyloid-beta for early hippocampal hyperactivity in a mouse model of Alzheimer's disease. Proceedings of the National Academy of Sciences of the United States of America 109, 8740-8745.

Busche, M.A., and Konnerth, A. (2016). Impairments of neural circuit function in Alzheimer's disease. Philosophical transactions of the Royal Society of London Series B, Biological sciences 371 . 
Cai, D.J., Aharoni, D., Shuman, T., Shobe, J., Biane, J., Song, W., Wei, B., Veshkini, M., La-Vu, M., Lou, J., et al. (2016). A shared neural ensemble links distinct contextual memories encoded close in time. Nature 534, 115-118.

Cembrowski, M.S., Phillips, M.G., DiLisio, S.F., Shields, B.C., Winnubst, J., Chandrashekar, J., Bas, E., and , $\leq \mu, \mu m S p r u s t o n, ~ N . ~(2018)$. Dissociable Structural and Functional Hippocampal Outputs via Distinct Subiculum Cell Classes. Cell 174, 1036.

Chen, T.W., Wardill, T.J., Sun, Y., Pulver, S.R., Renninger, S.L., Baohan, A., Schreiter, E.R., Kerr, R.A., Orger, M.B., Jayaraman, V., et al. (2013). Ultrasensitive fluorescent proteins for imaging neuronal activity. Nature 499, 295-300.

De Strooper, B., and Karran, E. (2016). The Cellular Phase of Alzheimer's Disease. Cell 164, 603-615.

Frere, S., and Slutsky, I. (2018). Alzheimer's Disease: From Firing Instability to Homeostasis Network Collapse. Neuron 97, 32-58.

Friedrich, J., Zhou, P., and Paninski, L. (2017). Fast online deconvolution of calcium imaging data. PLoS computational biology 13, e1005423.

Fuhrmann, F., Justus, D., Sosulina, L., Kaneko, H., Beutel, T., Friedrichs, D., Schoch, S., Schwarz, M.K., Fuhrmann, M., and Remy, S. (2015). Locomotion, Theta Oscillations, and the Speed-Correlated Firing of Hippocampal Neurons Are Controlled by a Medial Septal Glutamatergic Circuit. Neuron 86, 1253-1264.

Góis, Z.H.T.D., and Tort, A.B.L. (2018). Characterizing Speed Cells in the Rat Hippocampus. Cell Reports 25, 1872-1884.e1874.

Grieco, S.F., Qiao, X., Johnston, K.G., Chen, L., Nelson, R.R., Lai, C., Holmes, T.C., and Xu, X. (2021). Neuregulin signaling mediates the acute and sustained antidepressant effects of subanesthetic ketamine. Transl Psychiatry 11, 144. 
Grieco, S.F., Qiao, X., Zheng, X., Liu, Y., Chen, L., Zhang, H., Yu, Z., Gavornik, J.P., Lai, C., Gandhi, S.P., et al. (2020). Subanesthetic Ketamine Reactivates Adult Cortical Plasticity to Restore Vision from Amblyopia. Curr Biol 30, 3591-3603 e3598.

Grienberger, C., Rochefort, N.L., Adelsberger, H., Henning, H.A., Hill, D.N., Reichwald, J., Staufenbiel, M., and Konnerth, A. (2012). Staged decline of neuronal function in vivo in an animal model of Alzheimer's disease. Nature communications 3, 774.

Harris, S.S., Wolf, F., De Strooper, B., and Busche, M.A. (2020). Tipping the Scales: PeptideDependent Dysregulation of Neural Circuit Dynamics in Alzheimer's Disease. Neuron 107, 417-435.

Indersmitten, T., Schachter, M.J., Young, S., Welty, N., Otte, S., Nassi, J.J., Lovenberg, T., Bonaventure, P., and Wyatt, R.M. (2019). In vivo Calcium Imaging Reveals That Cortisol Treatment Reduces the Number of Place Cells in Thy1-GCaMP6f Transgenic Mice. Front Neurosci-Switz 13.

Jun, H., Bramian, A., Soma, S., Saito, T., Saido, T.C., and Igarashi, K.M. (2020). Disrupted Place Cell Remapping and Impaired Grid Cells in a Knockin Model of Alzheimer's Disease. Neuron 107, 1095-1112 e1096.

Jung, M.W., Wiener, S.I., and McNaughton, B.L. (1994). Comparison of spatial firing characteristics of units in dorsal and ventral hippocampus of the rat. J Neurosci 14,7347 7356.

Laird, N.M., and Ware, J.H. (1982). Random-effects models for longitudinal data. Biometrics 38, 963-974.

Lerdkrai, C., Asavapanumas, N., Brawek, B., Kovalchuk, Y., Mojtahedi, N., Olmedillas Del Moral, M., and Garaschuk, O. (2018). Intracellular $\mathrm{Ca}(2+)$ stores control in vivo neuronal hyperactivity in a mouse model of Alzheimer's disease. Proceedings of the National Academy of Sciences of the United States of America 115, E1279-E1288. 
Lever, C., Wills, T., Cacucci, F., Burgess, N., and O'Keefe, J. (2002). Long-term plasticity in hippocampal place-cell representation of environmental geometry. Nature 416, 90-94.

Mclean, R.A., Sanders, W.L., and Stroup, W.W. (1991). A Unified Approach to Mixed LinearModels. Am Stat 45, 54-64.

Mesulam, M.M. (2000). A plasticity-based theory of the pathogenesis of Alzheimer's disease. Annals of the New York Academy of Sciences 924, 42-52.

Naber, P.A., Witter, M.P., and Lopes Silva, F.H. (2000). Networks of the hippocampal memory system of the rat. The pivotal role of the subiculum. Annals of the New York Academy of Sciences 911, 392-403.

Oddo, S., Caccamo, A., Shepherd, J.D., Murphy, M.P., Golde, T.E., Kayed, R., Metherate, R., Mattson, M.P., Akbari, Y., and LaFerla, F.M. (2003). Triple-transgenic model of Alzheimer's disease with plaques and tangles: intracellular Abeta and synaptic dysfunction. Neuron 39, 409-421.

Palop, J.J., and Mucke, L. (2016). Network abnormalities and interneuron dysfunction in Alzheimer disease. Nature reviews Neuroscience 17, 777-792.

Pnevmatikakis, E.A., and Giovannucci, A. (2017). NoRMCorre: An online algorithm for piecewise rigid motion correction of calcium imaging data. Journal of neuroscience methods 291, 83-94.

Scheyltjens, I., Laramee, M.E., Van den Haute, C., Gijsbers, R., Debyser, Z., Baekelandt, V., Vreysen, S., and Arckens, L. (2015). Evaluation of the expression pattern of $r A A V 2 / 1,2 / 5$, $2 / 7,2 / 8$, and $2 / 9$ serotypes with different promoters in the mouse visual cortex. The Journal of comparative neurology 523, 2019-2042.

Selkoe, D.J. (2002). Alzheimer's disease is a synaptic failure. Science 298, 789-791.

Skaggs, W.E., McNaughton, B.L., Wilson, M.A., and Barnes, C.A. (1996). Theta phase precession in hippocampal neuronal populations and the compression of temporal sequences. Hippocampus 6, 149-172. 
Stimmell, A.C., Baglietto-Vargas, D., Moseley, S.C., Lapointe, V., Thompson, L.M., LaFerla, F.M., McNaughton, B.L., and Wilber, A.A. (2019). Impaired Spatial Reorientation in the 3xTg-AD Mouse Model of Alzheimer's Disease. Scientific reports 9, 1311.

Stobart, J.L., Ferrari, K.D., Barrett, M.J.P., Stobart, M.J., Looser, Z.J., Saab, A.S., and Weber, B. (2018). Long-term In Vivo Calcium Imaging of Astrocytes Reveals Distinct Cellular Compartment Responses to Sensory Stimulation. Cereb Cortex 28, 184-198.

Sun, Y., Jin, S., Lin, X., Chen, L., Qiao, X., Jiang, L., Zhou, P., Johnston, K.G., Golshani, P., Nie, Q., et al. (2019). CA1-projecting subiculum neurons facilitate object-place learning. Nature neuroscience 22, 1857-1870.

Weller, J., and Budson, A. (2018). Current understanding of Alzheimer's disease diagnosis and treatment. F1000Research 7.

Wong, J.C., Grieco, S.F., Dutt, K., Chen, L., Thelin, J.T., Inglis, G.A.S., Parvin, S., Garraway, S.M., Xu, X., Goldin, A.L., et al. (2021). Autistic-like behavior, spontaneous seizures, and increased neuronal excitability in a Scn8a mouse model. Neuropsychopharmacology.

Xu, X., Sun, Y., Holmes, T.C., and Lopez, A.J. (2016). Noncanonical connections between the subiculum and hippocampal CA1. The Journal of comparative neurology 524, 3666-3673.

Yang, X., Yao, C., Tian, T., Li, X., Yan, H., Wu, J., Li, H., Pei, L., Liu, D., Tian, Q., et al. (2018). A novel mechanism of memory loss in Alzheimer's disease mice via the degeneration of entorhinal-CA1 synapses. Molecular psychiatry 23, 199-210.

Zhang, S., Schonfeld, F., Wiskott, L., and Manahan-Vaughan, D. (2014). Spatial representations of place cells in darkness are supported by path integration and border information. Front Behav Neurosci 8, 222.

Zhou, P., Resendez, S.L., Rodriguez-Romaguera, J., Jimenez, J.C., Neufeld, S.Q., Giovannucci, A., Friedrich, J., Pnevmatikakis, E.A., Stuber, G.D., Hen, R., et al. (2018a). Efficient and accurate extraction of in vivo calcium signals from microendoscopic video data. eLife 7, e28728. 
Zhou, P., Resendez, S.L., Rodriguez-Romaguera, J., Jimenez, J.C., Neufeld, S.Q., Giovannucci, A., Friedrich, J., Pnevmatikakis, E.A., Stuber, G.D., Hen, R., et al. (2018b). Efficient and accurate extraction of in vivo calcium signals from microendoscopic video data. Elife 7.

Ziv, Y., Burns, L.D., Cocker, E.D., Hamel, E.O., Ghosh, K.K., Kitch, L.J., El Gamal, A., and Schnitzer, M.J. (2013). Long-term dynamics of CA1 hippocampal place codes. Nature neuroscience 16, 264-266. 


\section{Figure legends}

Figure 1. Age-dependent change in amyloid beta $(A \beta)$ and phospho-tau accumulation in 3xTg-AD mouse hippocampus. A. Comparison of $A \beta$ pathology in the 6.5-month-old and 18month-old 3xTg-AD and control, Non-Tg mice. 6.5-month-old 3xTg-AD mice show intraneuronal $A \beta$ staining in the pyramidal layer of hippocampal CA1 (left bottom panels). $A \beta$ staining is red and DAPI staining is blue. The accumulation of $A \beta$ (plaques, arrows) is distributed across all the layers of hippocampal CA1 in an 18-month-old 3xTg-AD mouse (bottom right panel) as compared to a non-transgenic control mouse (Non-Tg) (top right panel). B. Phospho-tau builds up in the 3xTg-AD mouse hippocampus. Tau staining is red. 6.5 months old 3xTg-AD (bottom left) mice shows sparsely tau labeling in distal CA1. The 18-month-old group exhibits elevated tau pathology in hippocampal CA1 pyramidal cells (arrows) (bottom right). The Non-Tg (top two panels) mouse hippocampus does not develop tau pathology. Scale bar $=200 \mu \mathrm{m}$.

Figure 2. In vivo neural calcium imaging of hippocampal CA1 excitatory neurons in 3xTgAD and Non-Tg mice. A. The scheme for viral injection and miniaturized fluorescent microscope (miniscope) recording in hippocampal CA1 region. A 1.8-mm diameter GRIN lens (shown in grey) was implanted at the same location as AAV1 injection site for in vivo calcium signal recording in the behavioral animals. B. A coronal section image shows the location for GRIN lens implantation in hippocampal CA1. DAPI staining is blue. GCaMP6f signal is green. Scale bar = $400 \mu \mathrm{m}$. Right panel: the higher magnification image of the left panel. The GCaMP6f infected cells are restricted to the pyramidal layers of the CA1 region. Scale bar $=40 \mu \mathrm{m}$. C. Examples of neuron footprints from CNMF-E extraction for data processing. Left to right, the example mice are from young-age Non-Tg, young-age 3xTg-, old-age Non-Tg and old-age 3xTg-AD, respectively. D. Extracted calcium traces. Top: Blue line represents calcium signal and the corresponding deconvoluted spiking activity is plotted by the red line. Bottom: the magnified calcium signals in the black box. E-H. 3xTg-AD CA1 cells exhibit less place-specific firing 
properties compared to Non-Tg CA1 cells during open field exploration. E. Calcium response and combined spatial rate map from a young age Non-Tg mouse. Left: Denoised calcium signal of one neuron. Middle: travel trajectory in the circular arena is plotted by black line. Red dots represent the locations in which the spike events are higher than threshold. Right: Rate maps of fluorescence firing rate. The arena is divided into $10 \times 10 \mathrm{~mm}$ bins and each bin's firing rate is calculated as the total number of spikes divided by the total time mice spend inside the bin. The rate map is smoothed with $100 \times 100 \mathrm{~mm}$ Gaussian kernel (standard deviation $=20 \mathrm{~mm}$ ). F, G, $\mathrm{H}$ are arranged in the same format for old-age Non-Tg, young-age 3xTg-AD and old-age 3xTgAD mice.

\section{Figure 3. 3xTg-AD CA1 cells exhibit altered calcium activities and impaired spatial coding during open field exploration.}

A. Violin plots of calcium event firing rates of all neurons from different groups of mice. Only the periods with movement speeds higher than $5 \mathrm{~mm} / \mathrm{sec}$ were used for firing rate calculation, and firing rates from trials within the same geometry were averaged. B. Violin plots of calcium event amplitudes of all neurons from different groups of mice. C. Cumulative distribution plots of spatial information scores (in bits/event) for all place cells from Non-Tg old, 3xTg-AD old, NonTg young and 3xTg-AD young mice exploring in both square and circle arenas. D. Cumulative distribution plots of sparsity of all neurons for all Non-Tg old, 3xTg-AD old, Non-Tg young and 3xTg-AD young mice in both square and circular arenas. E. Cumulative distribution plots of spatial coherence of all neurons for all Non-Tg old, 3xTg-AD old, Non-Tg young and 3xTg-AD young mice, in both square and circular arenas. LME analyses were used for A-B; two-sample Kolmogorov-Smirnov tests were used for C-E. ${ }^{*},{ }^{* *}$ and ${ }^{* * *}$ indicate the significance levels with the respective $p$ values of $<0.05,<0.005$, and $<0.0005$. 
Figure 4. Linear track experiment data also support impaired spatial coding in 3xTg-AD hippocampal CA1. A-D. Response of example neurons running on a horizontal linear track from mice with different genotypes and ages. Top: the black line represents the trajectory of mice. Superimposed red dots represent the locations where calcium events occur. Bottom: smoothed spatial rate maps. E. Violin plots of calcium event firing rates of all neurons from different groups of mice. Only the periods with movement speeds higher than $5 \mathrm{~mm} / \mathrm{sec}$ were used for firing rate calculation, and firing rates from trials with the same track direction were averaged. F. Violin plots of calcium event amplitudes of all neurons from different groups of mice. G. Cumulative distribution plots of spatial information score (in bits/event) for all place cells from Non-Tg old, 3xTg-AD old, Non-Tg young and 3xTg-AD young mice, for both horizontal and vertical tracks in two directions. I. Cumulative distribution plots of spatial coherence of all neurons for Non-Tg old, 3xTg-AD old, Non-Tg young and 3xTg-AD young mice, in both square and circle arena. LME analyses were used for E-F; two-sample KolmogorovSmirnov tests were used for G-I. * * ** and *** indicate the significance levels with the respective $p$ values of $<0.05,<0.005$, and $<0.0005$.

Figure 5. Neural calcium activities of 3xTg-AD mice exhibit stronger locomotion modulation than Non-Tg mice. A. Raster plots of calcium activities of hundreds of CA1 cells along with time (top), movement epochs (middle) and comparison between the z-scored ensemble calcium trace and movement speed (bottom, ensemble calcium trace: orange; velocity trace: blue). The high-speed epoch is shown as the black pattern at the time points where the movement speed is higher than the speed threshold, which is defined as the averaged median movement speed of the group. Four example mice of Non- $\mathrm{Tg}$ young, 3xTgAD young, Non-Tg old and 3xTg-AD old, respectively, are shown. B. Distribution of mean 
movement speeds of different groups of mice. Each dot represents the mean speed of one mouse in one trial (circle or square). C. Cumulative distribution plots of correlation values between moving speed and all individual neuron's calcium response. Correlation values from trials with the same geometry are averaged, and each dot represents a single neuron in one type of trial (square or circle). D. Cumulative distribution plots of correlation values between moving speed and all place cells' calcium trace. Two-sample Kolmogorov-Smirnov tests were used for C-D. ${ }^{*},{ }^{* *}$ and ${ }^{* * *}$ indicate the significance levels with the respective $p$ values of $<0.05$, $<0.005$, and $<0.0005$. 


\section{Figures}

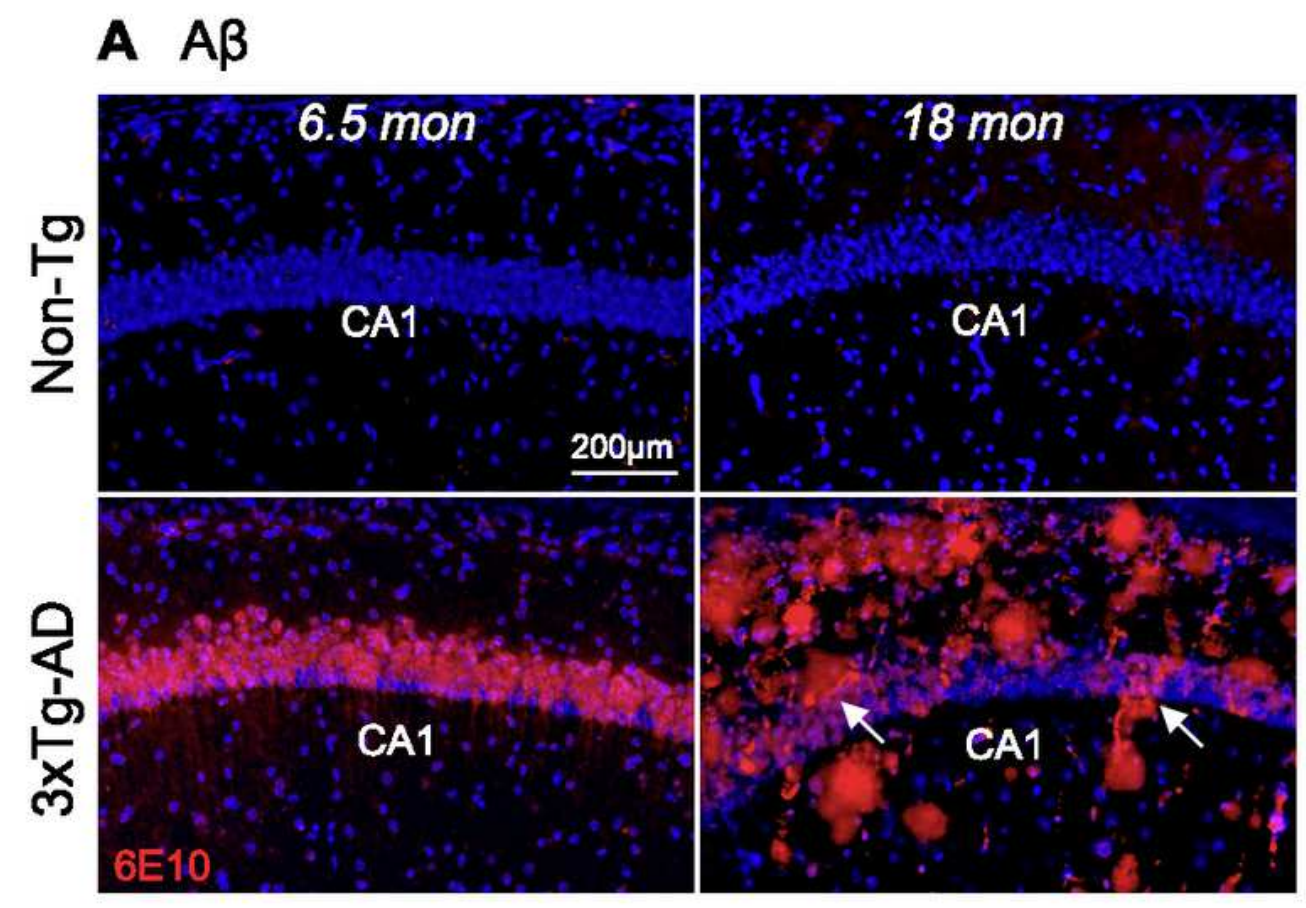

\section{B Tau}

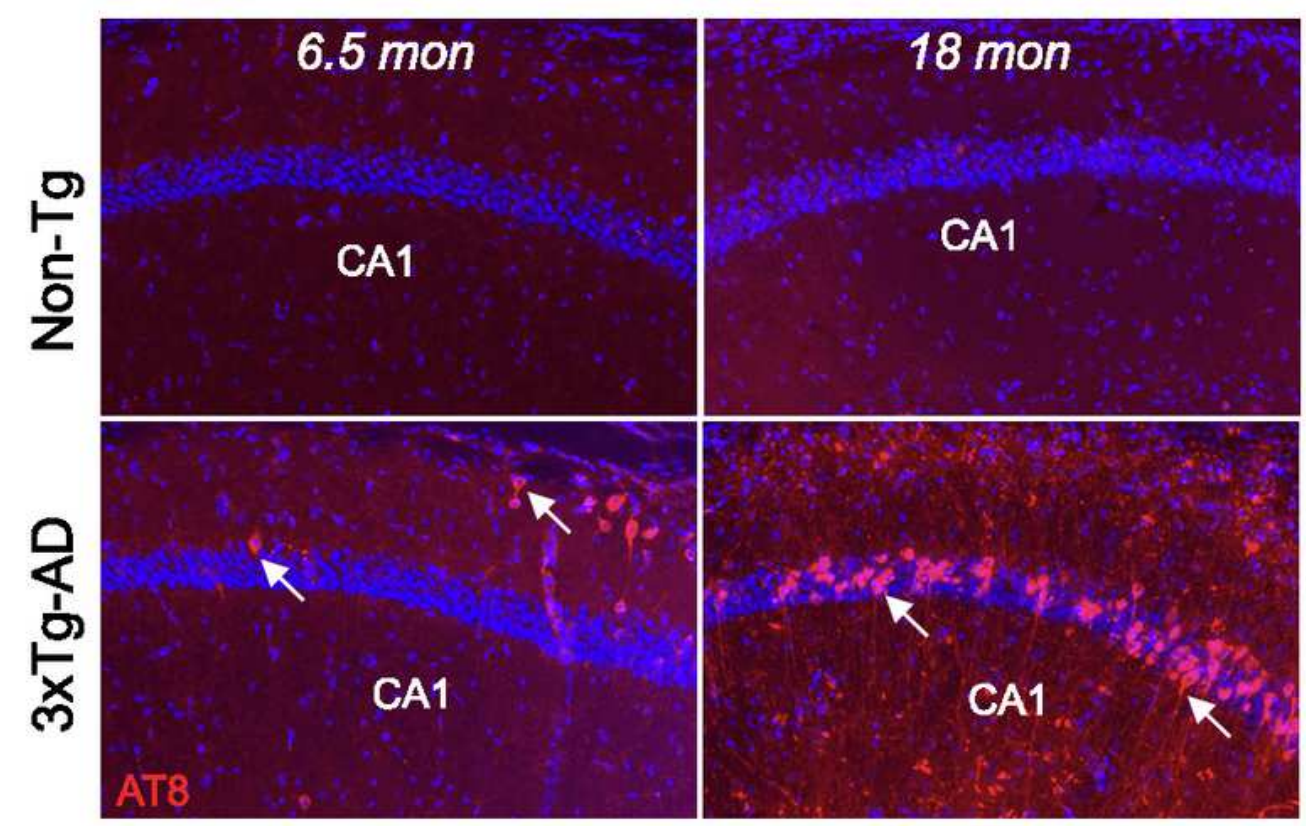

Figure 1

Age-dependent change in amyloid beta $(A \beta)$ and phospho-tau accumulation in 3xTg-AD mouse hippocampus. A. Comparison of $A \beta$ pathology in the 6.5-month-old and 18-month-old 3xTg-AD and control, Non-Tg mice. 6.5-month-old 3xTg-AD mice show intraneuronal $A \beta$ staining in the pyramidal layer 
of hippocampal CA1 (left bottom panels). A $\beta$ staining is red and DAPI staining is blue. The accumulation of $A \beta$ (plaques, arrows) is distributed across all the layers of hippocampal CA1 in an 18-month-old 3xTg$A D$ mouse (bottom right panel) as compared to a non-transgenic control mouse (Non-Tg) (top right panel). B. Phospho-tau builds up in the 3xTg-AD mouse hippocampus. Tau staining is red. 6.5 months old 3xTg-AD (bottom left) mice shows sparsely tau labeling in distal CA1. The 18-month-old group exhibits elevated tau pathology in hippocampal CA1 pyramidal cells (arrows) (bottom right). The Non-Tg (top two panels) mouse hippocampus does not develop tau pathology. Scale bar $=200 \mu \mathrm{m}$.

A
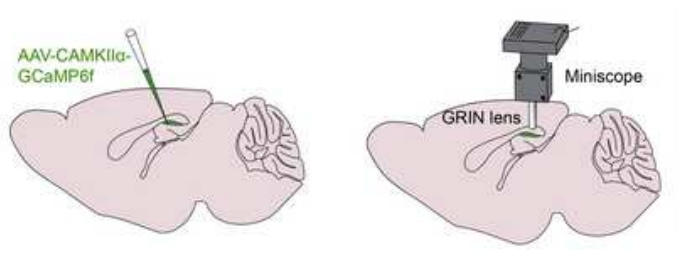

B
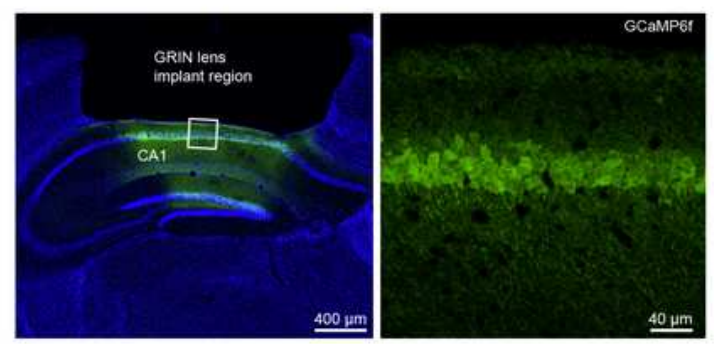

C
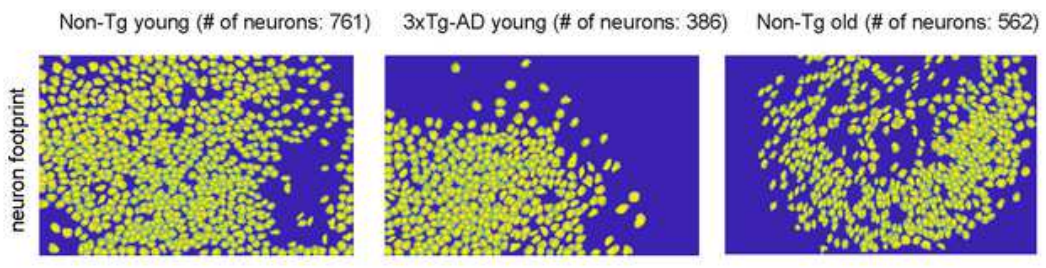

3xTtg-AD old (\# of neurons: 264 )

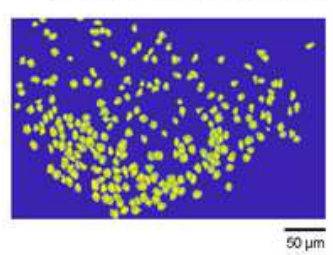

D

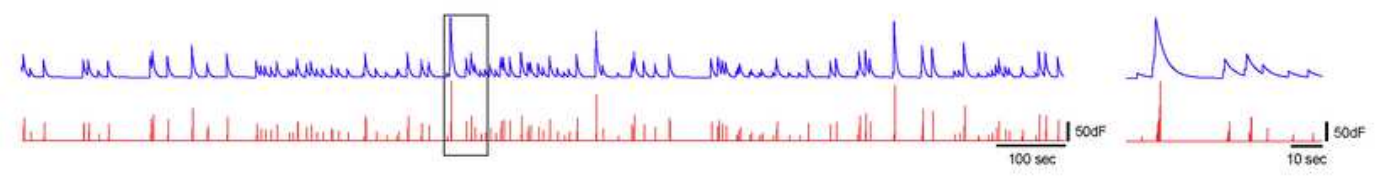

E

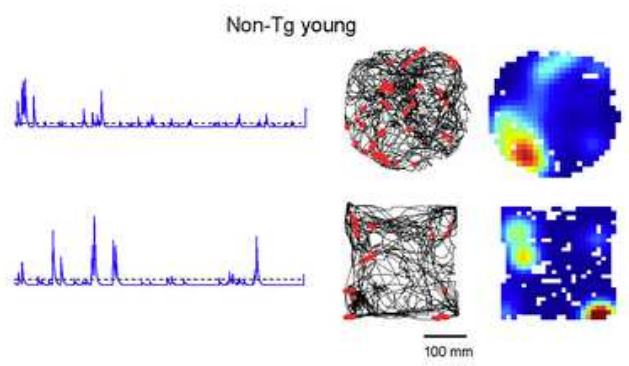

Non-Tg old
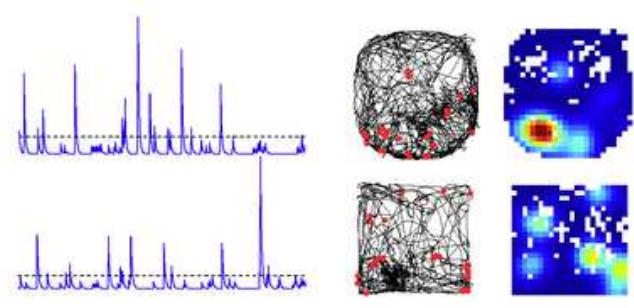

F

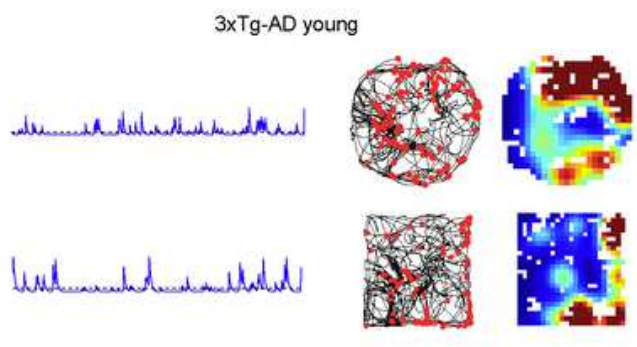

H

$3 \times T g-A D$ old

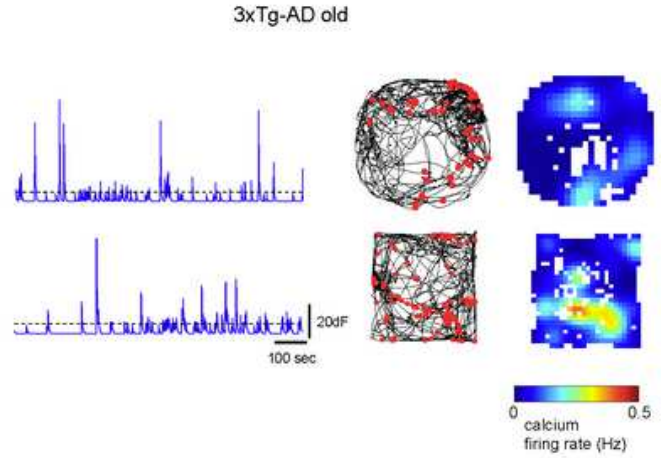




\section{Figure 2}

In vivo neural calcium imaging of hippocampal CA1 excitatory neurons in 3xTg-AD and Non-Tg mice. A. The scheme for viral injection and miniaturized fluorescent microscope (miniscope) recording in hippocampal CA1 region. A 1.8-mm diameter GRIN lens (shown in grey) was implanted the same location as AAV1 injection site for in vivo calcium signal recording in the behavioral animals. B. A coronal section image shows the location for GRIN lens implantation in hippocampal CA1. DAPI staining is blue. GCaMP6f signal is green. Scale bar $=400 \mu \mathrm{m}$. Right panel: the higher magnification image of the left panel. The GCaMP6f infected cells are restricted to the pyramidal layers of the CA1 region. Scale bar $=40$ $\mu \mathrm{m}$. C. Examples of neuron footprints from CNMF-E extraction for data processing. Left to right, the example mice are from young-age Non-Tg, young-age 3xTg-, old-age Non-Tg and old-age 3xTg-AD, respectively. D. Extracted calcium traces. Top: Blue line represents calcium signal and the corresponding deconvoluted spiking activity is plotted by the red line. Bottom: the magnified calcium signals in the black box. E-H. 3xTg-AD CA1 cells exhibit less place-specific firing properties compared to Non-Tg CA1 cells during open field exploration. E. Calcium response and combined spatial rate map from a young age NonTg mouse. Left: Denoised calcium signal of one neuron. Middle: travel trajectory in the circular arena is plotted by black line. Red dots represent the locations in which the spike events are higher than threshold. Right: Rate maps of fluorescence firing rate. The arena is divided into $10 \times 10 \mathrm{~mm}$ bins and each bin's firing rate is calculated as the total number of spikes divided by the total time mice spend inside the bin. The rate map is smoothed with $100 \times 100 \mathrm{~mm}$ Gaussian kernel (standard deviation $=20 \mathrm{~mm}$ ). F, G, H are arranged in the same format for old-age Non-Tg, young-age 3xTg-AD and old-age 3xTg-AD mice. 

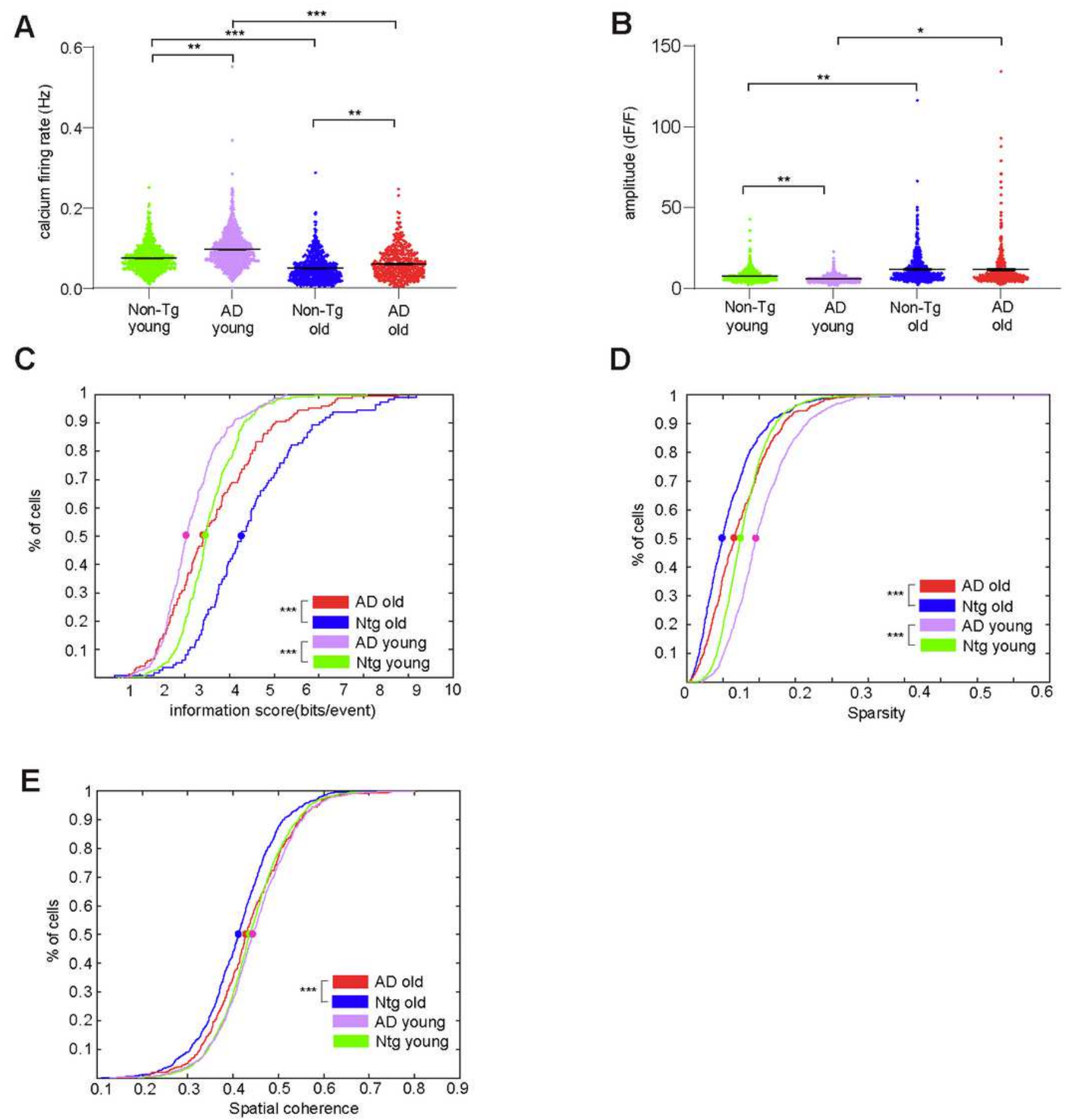

\section{Figure 3}

3xTg-AD CA1 cells exhibit altered calcium activities and impaired spatial coding during open field exploration. A. Violin plots of calcium event firing rates of all neurons from different groups of mice. Only the periods with movement speeds higher than $5 \mathrm{~mm} / \mathrm{sec}$ were used for firing rate calculation, and firing rates from trials within the same geometry were averaged. B. Violin plots of calcium event amplitudes of all neurons from different groups of mice. C. Cumulative distribution plots of spatial information scores 
(in bits/event) for all place cells from Non-Tg old, 3xTg-AD old, Non-Tg young and 3xTg-AD young mice exploring in both square and circle arenas. D. Cumulative distribution plots of sparsity of all neurons for all Non-Tg old, 3xTg-AD old, Non-Tg young and 3xTg-AD young mice in both square and circular arenas. E. Cumulative distribution plots of spatial coherence of all neurons for all Non-Tg old, 3xTg-AD old, NonTg young and 3xTg-AD young mice, in both square and circular arenas. LME analyses were used for A-B; two-sample Kolmogorov-Smirnov tests were used for $\mathrm{C}-\mathrm{E}$. * ${ }^{*}$ * and $* \star \star$ indicate the significance levels with the respective $p$ values of $<0.05,<0.005$, and $<0.0005$.

A

A

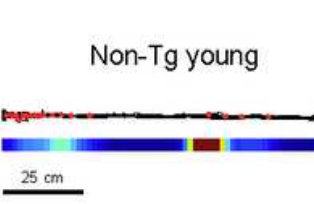

B

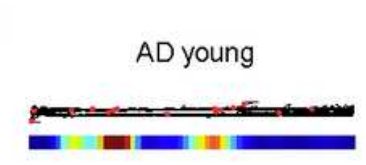

C

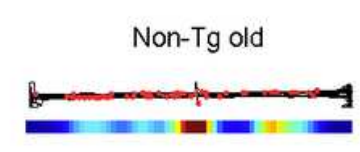

D

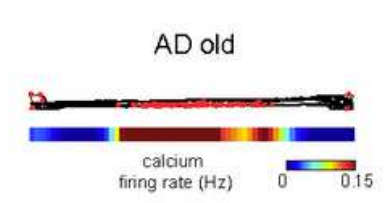

E
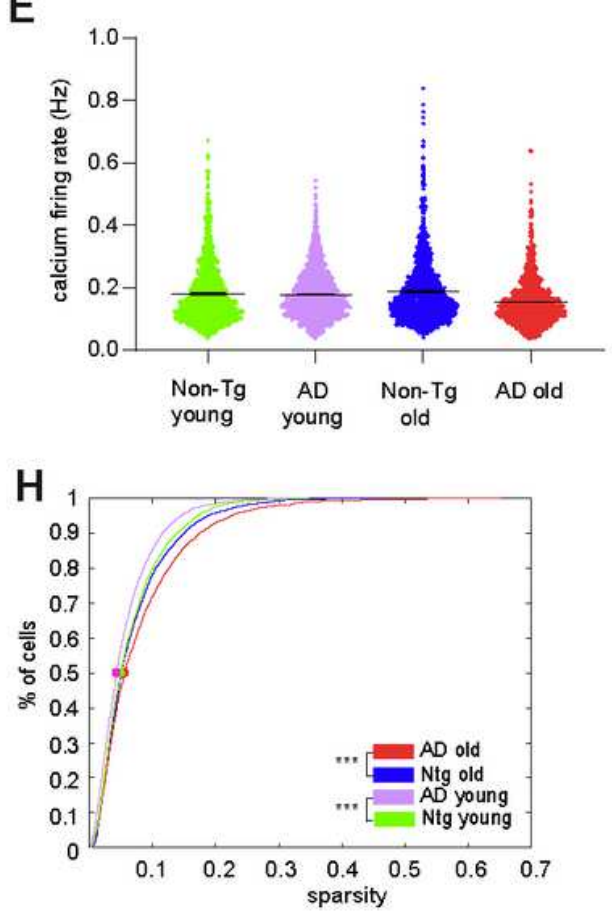

F
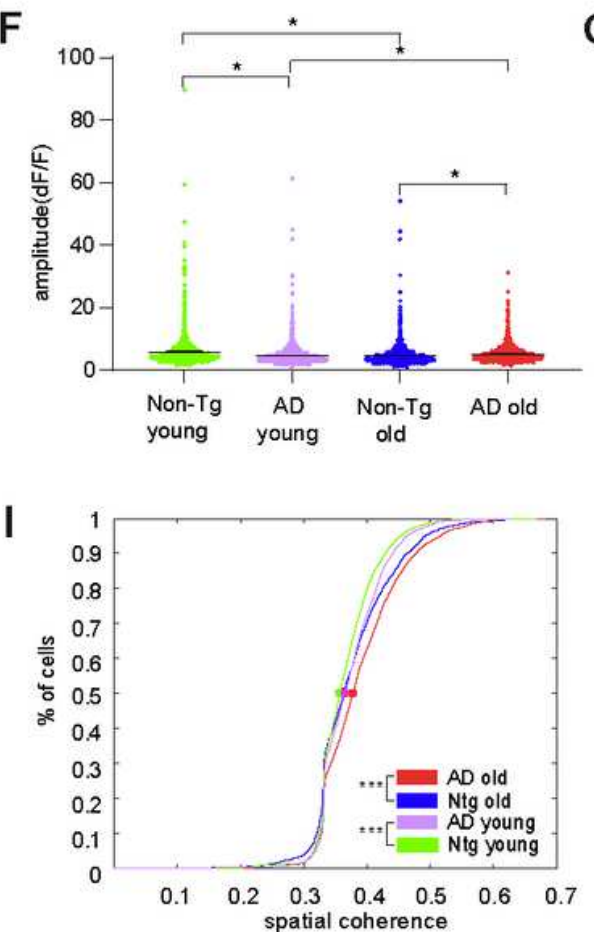

G
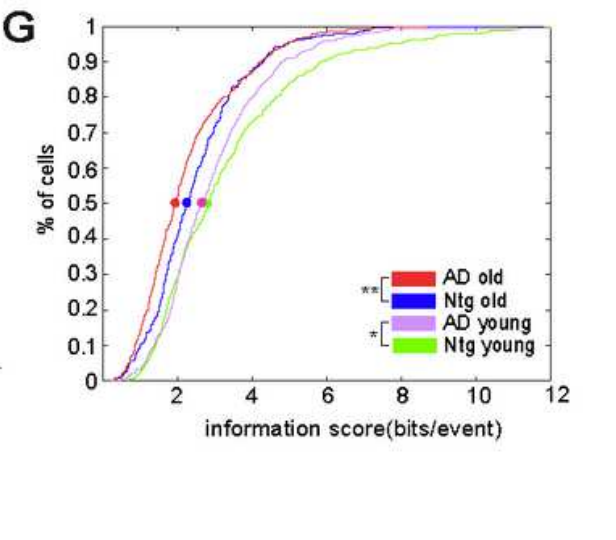
tracks in two directions. I. Cumulative distribution plots of spatial coherence of all neurons for Non-Tg old, $3 x T g-A D$ old, Non-Tg young and 3xTg-AD young mice, in both square and circle arena. LME analyses were used for E-F; two-sample Kolmogorov-Smirnov tests were used for G-I. *, ** and *** indicate the significance levels with the respective $p$ values of $<0.05,<0.005$, and $<0.0005$.

A

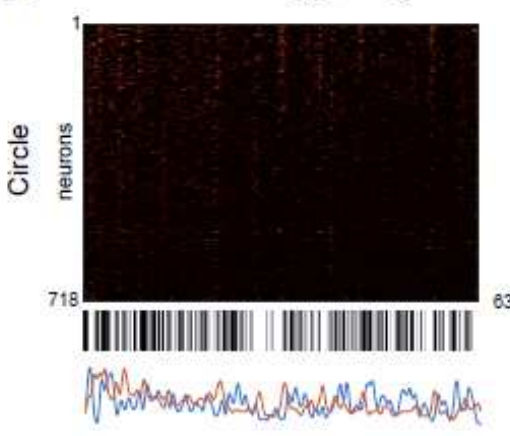

AD young
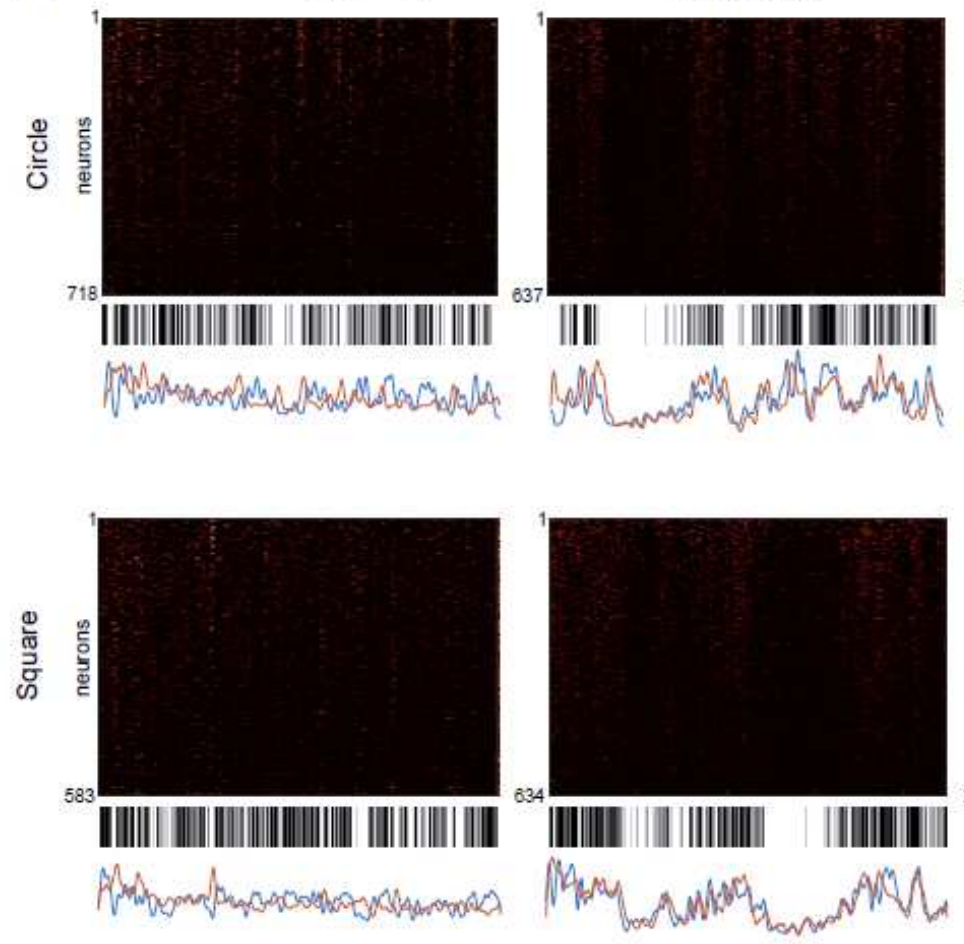

$\mathrm{AD}$ old

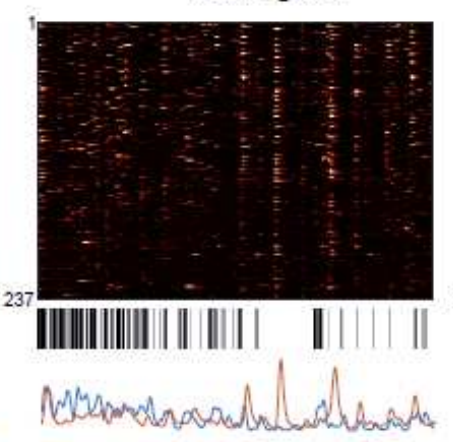

- zscored speed (smoothed)

- zscored ensemble calcium trace (smoothed)
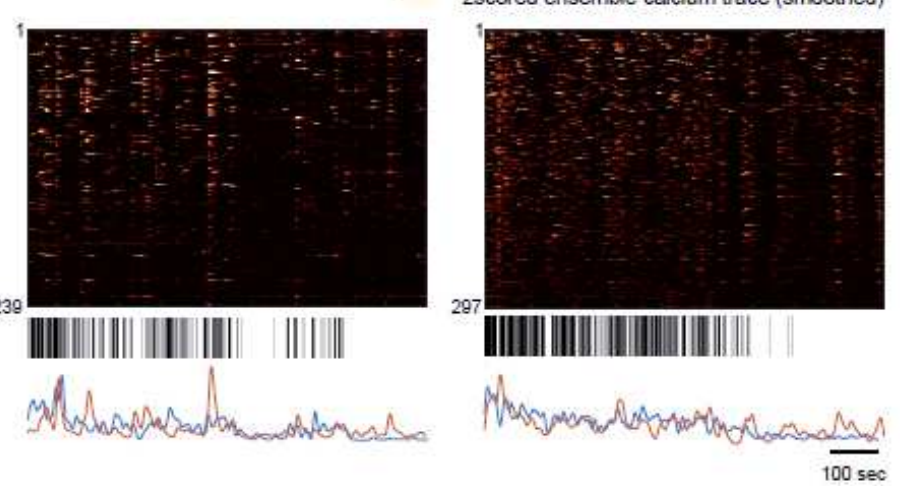
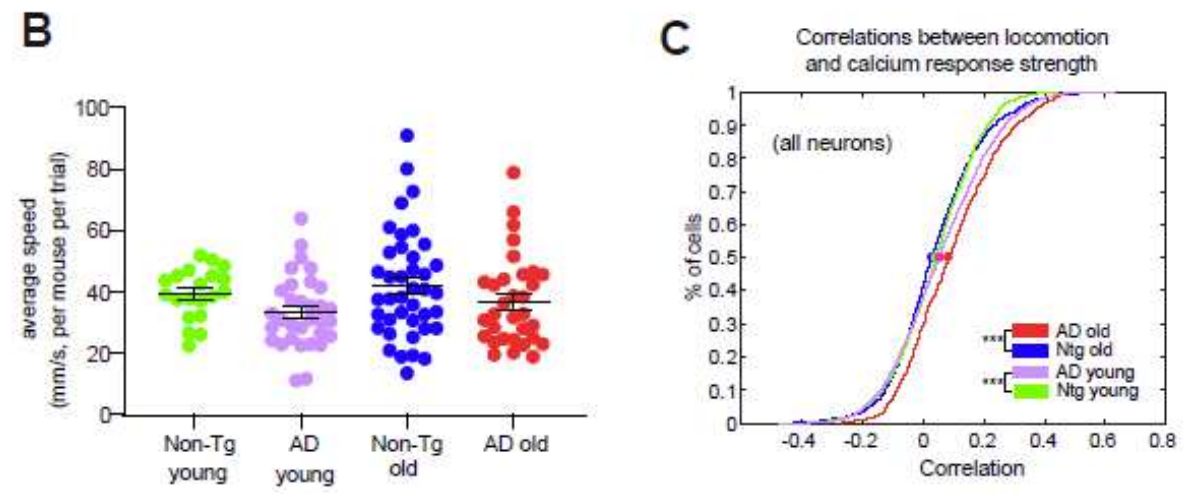

D

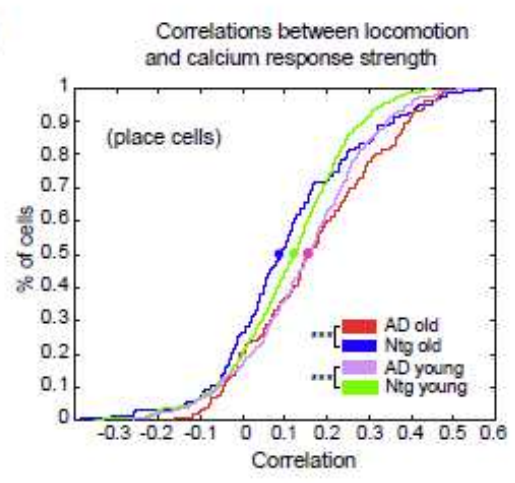

\section{Figure 5}

Neural calcium activities of 3xTg-AD mice exhibit stronger locomotion modulation than Non-Tg mice. A. Raster plots of calcium activities of hundreds of CA1 cells along with time (top), movement epochs (middle) and comparison between the z-scored ensemble calcium trace and movement speed (bottom, ensemble calcium trace: orange; velocity trace: blue). The high-speed epoch is shown as the black pattern at the time points where the movement speed is higher than the speed threshold, which is defined as the averaged median movement speed of the group. Four example mice of Non-Tg young, 3xTg-AD young, Non-Tg old and 3xTg-AD old, respectively, are shown. B. Distribution of mean movement speeds of 
different groups of mice. Each dot represents the mean speed of one mouse in one trial (circle or square). C. Cumulative distribution plots of correlation values between moving speed and all individual neuron's calcium response. Correlation values from trials with the same geometry are averaged, and each dot represents a single neuron in one type of trial (square or circle). D. Cumulative distribution plots of correlation values between moving speed and all place cells' calcium trace. Two-sample KolmogorovSmirnov tests were used for C-D. *, ${ }^{*}$ and ${ }^{* \star *}$ indicate the significance levels with the respective $p$ values of $<0.05,<0.005$, and $<0.0005$. 